\title{
Fluid-elastic structure interaction simulation by using ordinary state-based peridynamics and peridynamic differential operator
}

\author{
Yan Gao and Selda Oterkus* \\ Department of Naval Architecture, Ocean and Marine Engineering \\ University of Strathclyde, 100 Montrose Street, Glasgow, G4 0LZ, United Kingdom \\ *Corresponding author: selda.oterkus@strath.ac.uk
}

\begin{abstract}
The fluid-structure interaction phenomenon is often encountered in the ocean engineering field. In the present work, a non-local numerical model is developed for the simulations of weakly compressible viscous fluid and elastic structure interactions. The peridynamic theory is adopted for both the structure and fluid modelling. The elastic structure is described by using the ordinary state-based peridynamics, while the fluid is modelled by utilizing the peridynamic differential operator. Furthermore, the updated Lagrangian description is adopted for the fluid including the relative deformation gradient expressed by the peridynamic differential operator. The fluid-structure interface and its normal direction are calculated via the gradient of a colour function, which varies with the fluid motion and structure deformation. Besides, the interaction force exerted from fluid to structure is constrained to be always perpendicular to the moving interface. Hence the fluid motion and structural deformation are predicted simultaneously. The validation of the developed model is conducted through the simulation of a water dam break with a rubber gate. The good agreement between the peridynamic and the experiment results demonstrates the capability of the current model for solving fluid-elastic structure interaction problems.
\end{abstract}

Key Words: ordinary state-based peridynamics, peridynamic differential operator, non-local, fluid-structure interaction, dam break

\section{Introduction}

Fluid-structure interaction (FSI) is a class of problems with mutual dependence between the fluid and structural mechanics parts [1]. The FSI phenomenon widely exists in the 
engineering field, e.g. liquid sloshing [2], slamming [3], etc.. Therefore, it has been extensively and intensively studied for years.

There are mainly two types of simulation methods for FSI problems, i.e. simultaneous methods and partitioned methods. When the coupling between the fluid and structure is strong, or the fluid motion and structure deformation are comparable, it is convenient to employ a simultaneous solution [4]. One popular method is the Arbitrary-Lagrangian-Eulerian (ALE) formulation for coupling the fluid described by Eulerian formulation and the structure described by Lagrangian formulation [5]. The immersed boundary method couples the Eulerian fluid and the Lagrangian structure via interpolation in a bi-directional way [6]. Another promising solution falls into the category of fully Lagrangian description in which both the fluid and the structure are formulated in Lagrangian form. Taking the smoothing particle hydrodynamics (SPH) as an example, Antoci et al. [4] develop a coupled SPH-SPH model to simulate the behaviour of fluid and elastic structure by their SPH form of governing equations. The SPH-SPH model predicted results are compared with the ones obtained by the experiment, achieving a good agreement. A multiphase SPH model has been employed by Ruben Paredes and Len Imas [7] to solve the fluid-structure interaction problems. The simulations of the sloshing problems and the elastic gate problem are conducted to verify their SPH model. Furthermore, a complex fluid-structure dam-breaking problem is studied in [8] by using the multi-phase SPH method coupled with Adaptive-Particle-Refinement (APR) technique. An enhanced incompressible SPH-SPH coupled method is proposed by Khayyer et al. [9] to simulate the incompressible fluid and elastic structure interactions. The divergence-free property of the fluid velocity field is guaranteed by solving the Poisson Pressure Equation (PPE). Besides, a dynamic stabilizer technique is employed [10] to overcome the tension instability issues. The state-of-art development of the SPH-SPH or SPH coupled with other methods for FSI problems is reviewed by Moubiu Liu and Zhiliang Zhang [11]. For example, the discrete element method (DEM) can be coupled with the SPH method for solving the FSI problems, where DEM is used for the structure modelling while SPH is adopted for the fluid modelling [12]. In the study of Wu et al. [12], the free surface flow, as well as the structural failure, is considered. Moving particle semi-implicit (MPS) method is also utilized for the FSI problems. A multi-resolution MPS model is developed by Khayyer et al. [13] for the incompressible fluid-elastic structure interaction simulations. In their study, the problem of sloshing with an elastic baffle is simulated. The good agreement between the numerical results and the experiment results validates the accuracy of their model. Furthermore, an MPS model 
considering a dynamic equation of angular momentum conservation is proposed in [14] for FSI problems corresponding to the incompressible fluid flow and elastic structures.

Peridynamics (PD), which is a mesh-free method proposed by Silling [15], has been widely applied to solid fracture problems. Instead of using the partial differential form of the equation of motion in classical continuum mechanics, an integral to space and differential to time formulation is adopted in PD to predict the solid deformation. Hence, the equation of motion in the PD form will be valid everywhere regardless of discontinuities. As a result, the PD theory has drawn many attentions since its appearance. The simplest form of PD is the socalled bond-based PD. Since the shear deformation and bulk deformation are not distinguished, there is a Poisson's ratio constraint on the material property. The Poisson's ratio is fixed as 1/3 for two dimensional (2D) problems and 1/4 for three dimensional (3D) isotropic problems [16]. Consequently, non-ordinary state-based [17] and ordinary state based [16] peridynamic formulations are developed to overcome the Poisson's ratio limitations. The PD theory has been mainly applied for predicting damages in structures due to various loading conditions [1832]. In fluid structure interaction problems, capability of numerical models is required for predicting damages. Since no additional failure criterion is needed, the PD theory is suitable for modelling fluid structure interaction problems especially when structural failure is a concern. In this study, ordinary state-based peridynamic theory (OSB-PD) is adopted to simulate the elastic structure, so that the structural failure can be incorporated in the present model.

In light of the idea of PD theory, peridynamic differential operator (PDDO) is proposed by Madenci et al. [33]. Based on the Taylor series expansion and orthogonal function property, the PDDO can convert any order derivatives into their integral forms with user-defined order of accuracy. Furthermore, the material property parameters can be directly used in the PDDO integration, avoiding the derivation of PD parameters. In the authors' previous work, the PDDO has been employed to simulate the laminar fluid flow [34] and fluid flow coupled with heat transfer [35]. In the present study, PDDO is thus utilized for fluid flow modelling.

Since the PD theory is initially proposed for solid mechanics, the published PD models for FSI problems are limited. Coupled PD methods are developed by Oterkus et al [36], Ouichi et al.[37] and Barba et al. [38] that are capable of predicting hydraulic fracturing problems. Liu et al. [39] coupled PD with updated Lagrangian particle hydrodynamics to simulate icewater interactions. The PD theory is also used for ice modelling by including the ice breakage 
[40-42]. The updated Lagrangian particle hydrodynamics (ULPH) proposed by Tu et al. [43] is utilized to simulate the fluid flow.

In the literature, the use of PDDO on crack propagation simulations is limited. On the other hand, there is an extensive literature on PD applications in predicting damages in various structures, while its application on fluid mechanics is limited. Therefore, in this study, OSBPD [16] is used for the elastic deformation in the structure and PDDO [33] is used for the fluid flow simulations $[34,35]$. As a result, the formulation for elastic deformation does not have Poisson ratio limitation and has damage prediction capability [16]. At the same time, the use of the non-local fluid governing equations can achieve higher-order accuracy by using PDDO $[34,35]$. The comparison between different mesh free methods is also provided in Table 1.

Table 1 Comparison between different mesh free methods

\begin{tabular}{|c|c|c|c|c|}
\hline $\begin{array}{l}\text { Meshfree } \\
\text { methods }\end{array}$ & $\begin{array}{c}\text { Time } \\
\text { stepping } \\
\text { scheme } \\
\text { preferred }\end{array}$ & $\begin{array}{c}\text { Main } \\
\text { application } \\
\text { fields }\end{array}$ & Advantage & Disadvantage \\
\hline
\end{tabular}

$$
\begin{aligned}
& \text { 1) a nonlocal } \\
& \text { extension of } \\
& \text { continuum }
\end{aligned}
$$

Explicit

Fracture in mechanics

PD

[15] or Implicit Solids [15], damage in Multiphysics problems[45] formulation [15]

2) easy to implement for damage prediction capability [15]

Explicit [46] or SPH Implicit (ISPH) Fluid flow [46] Simple formulation [46]
Heavily dependent on choice of kernel function[48] 


\begin{tabular}{|c|c|c|c|c|}
\hline DEM & $\begin{array}{c}\text { Explicit } \\
\text { [49] }\end{array}$ & $\begin{array}{c}\text { Fracture in } \\
\text { Solids [49], } \\
\text { granular flow } \\
\text { [50] }\end{array}$ & $\begin{array}{l}\text { Models micro- } \\
\text { mechanical } \\
\text { interactions[51] }\end{array}$ & $\begin{array}{c}\text { Computationally } \\
\text { expensive [52] }\end{array}$ \\
\hline MPS & $\begin{array}{c}\text { Implicitly } \\
\text { calculates } \\
\text { pressure } \\
\text { [53] }\end{array}$ & $\begin{array}{c}\text { Incompressib } \\
\text { le Fluid flow } \\
\text { [53], nuclear } \\
\text { engineering } \\
{[54]}\end{array}$ & $\begin{array}{l}\text { Models } \\
\text { incompressible } \\
\text { fluid flow [53] }\end{array}$ & $\begin{array}{c}\text { Unphysical pressure } \\
\text { fluctuations [55] }\end{array}$ \\
\hline
\end{tabular}

The remainder of the paper is organized as follows. Section 2 and 3 provide the PD models for mechanical deformation and fluid flow, respectively. Section 4 provides the numerical implementations for fluid-structure simulations and emphasizes the coupling scheme between the fluid and the elastic structure. Section 5 presents the FSI numerical simulation, followed by the conclusion drawn in Section 6.

\section{PD Model for mechanical deformations}

In the present study, the ordinary state-based peridynamic theory (OSB-PD) is used for the elastic structure modelling as proposed by Silling $[15,16]$. The 2D equation of motion for point $\mathbf{x}_{s}$ in OSB-PD form is expressed as $[15,16]$

$$
\rho_{s} \ddot{\mathbf{u}}_{s}\left(\mathbf{x}_{s}, t\right)=\int_{H_{\mathbf{x}_{s}}}\left(\frac{2 \delta_{s} a d \Lambda}{\left|\mathbf{x}_{s}^{\prime}-\mathbf{x}_{s}\right|}\left(\theta_{s}+\theta_{s}^{\prime}\right)+4 \delta_{s} b s\right)\left(\frac{\mathbf{y}_{s}^{\prime}-\mathbf{y}_{s}}{\left|\mathbf{y}_{s}^{\prime}-\mathbf{y}_{s}\right|}\right) \mathrm{d} V_{s}^{\prime}+\mathbf{b}_{s}\left(\mathbf{x}_{s}, t\right)
$$

where $\rho_{s}$ represents the density of solid, $\ddot{\mathbf{u}}_{s}$ represents the acceleration of solid, $\delta_{s}$ represents the horizon size, and $\mathbf{b}_{s}$ represents the volumetric body force. The term $s$ is the PD bond stretch, $\Lambda$ is an auxiliary parameter, and $V_{s}^{\prime}$ is the volume of a material point $\mathbf{x}_{s}^{\prime}$ which is located within the horizon. As shown in Fig. 1, the parameters $\mathbf{y}_{s}$ and $\mathbf{y}_{s}^{\prime}$ are the updated positions. The parameters $\mathbf{x}_{s}$ and $\mathbf{x}_{s}^{\prime}$ are the initial positions. The peridynamic parameters $a$, $b$, and $d$ for 2D are defined as [16]

$$
a=\frac{1}{2}\left(K_{\theta}-2 \mu_{s}\right), b=\frac{6 \mu_{s}}{\pi h_{\text {thick }} \delta_{s}^{4}}, d=\frac{2}{\pi h_{\text {thick }} \delta_{s}^{3}}
$$


where $K_{\theta}$ is the bulk modulus, $\mu_{s}$ is the Lamé's second parameter, and $h_{\text {thick }}$ is the thickness. In Eq.(1) $\theta_{s}$ and $\theta_{s}^{\prime}$ are the dilatations of point $\mathbf{x}_{s}$ and $\mathbf{x}_{s}^{\prime}$, respectively. They are defined as [16]

$$
\theta_{s}=\int_{H_{\mathbf{x}_{s}}}\left(d \delta_{s} s \Lambda\right) \mathrm{d} V_{s}^{\prime}, \theta_{s}^{\prime}=\int_{H_{\mathbf{x}_{s}}}\left(d \delta_{s} s \Lambda\right) \mathrm{d} V_{s}
$$

with

$$
\begin{gathered}
s=\frac{\left|\mathbf{y}_{s}^{\prime}-\mathbf{y}_{s}\right|-\left|\mathbf{x}_{s}^{\prime}-\mathbf{x}_{s}\right|}{\left|\mathbf{x}_{s}^{\prime}-\mathbf{x}_{s}\right|}, \quad\left|\mathbf{y}_{s}^{\prime}-\mathbf{y}_{s}\right|=\mathbf{x}_{s}^{\prime}+\mathbf{u}_{s}^{\prime}-\mathbf{x}_{s}-\mathbf{u}_{s} \\
\Lambda=\left(\frac{\mathbf{y}_{s}^{\prime}-\mathbf{y}_{s}}{\left|\mathbf{y}_{s}^{\prime}-\mathbf{y}_{s}\right|}\right) \cdot\left(\frac{\mathbf{x}_{s}^{\prime}-\mathbf{x}_{s}}{\left|\mathbf{x}_{s}^{\prime}-\mathbf{x}_{s}\right|}\right)
\end{gathered}
$$

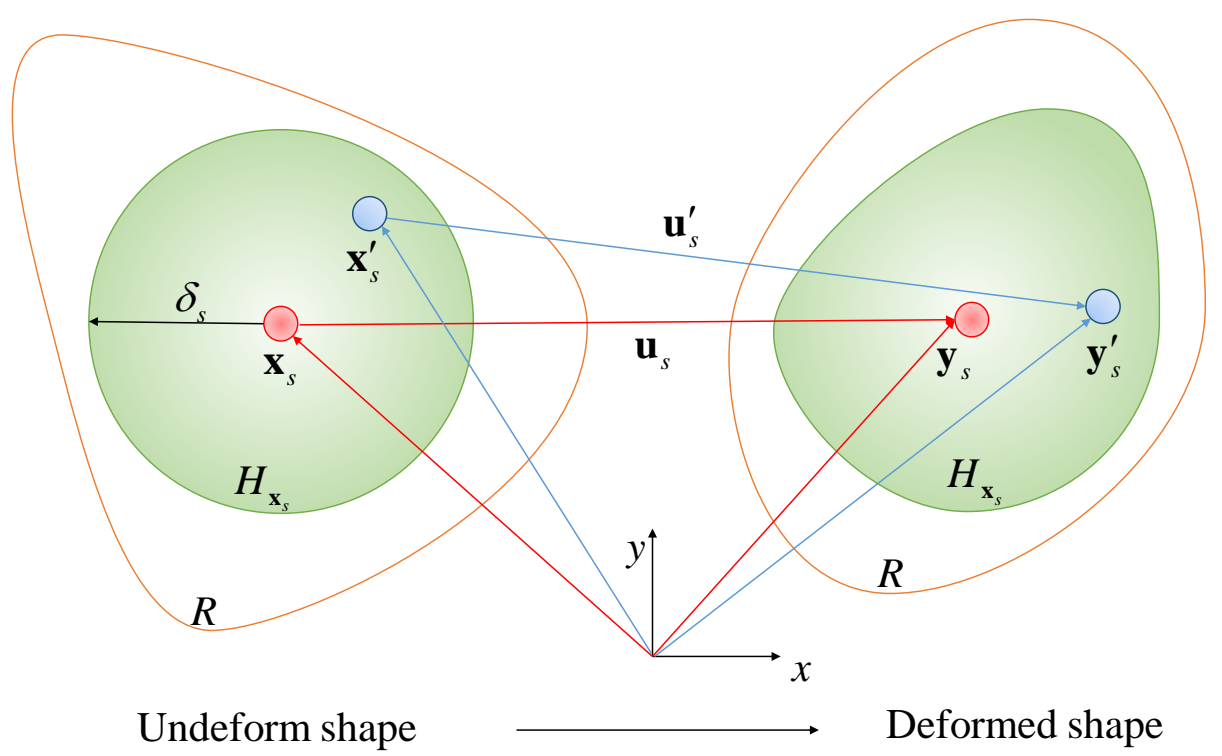

Fig. 1 Peridynamic interaction between the central point $\mathbf{X}_{s}$ and its family member $\mathbf{x}_{s}^{\prime}$ for two-dimensional space

\section{PDDO Model for fluid flow}

\subsection{PDDO definitions}

Inspired by the peridynamic theory, the spatial partial differential equations can be converted into their integral forms by using the peridynamic differential operator as proposed by Madenci et al. [33]. The PDDO form of differential equations up to second order is presented as [24] 


$$
\left\{\begin{array}{c}
\frac{\partial f(\mathbf{x})}{\partial x} \\
\frac{\partial f(\mathbf{x})}{\partial y} \\
\frac{\partial^{2} f(\mathbf{x})}{\partial x^{2}} \\
\frac{\partial^{2} f(\mathbf{x})}{\partial y^{2}} \\
\frac{\partial^{2} f(\mathbf{x})}{\partial x \partial y}
\end{array}\right\}=\int_{H_{\mathbf{x}}}\left(f\left(\mathbf{x}^{\prime}\right)-f(\mathbf{x})\right)\left\{\begin{array}{l}
g^{10}\left(\left|\mathbf{x}^{\prime}-\mathbf{x}\right|\right) \\
g^{01}\left(\left|\mathbf{x}^{\prime}-\mathbf{x}\right|\right) \\
g^{20}\left(\left|\mathbf{x}^{\prime}-\mathbf{x}\right|\right) \\
g^{02}\left(\left|\mathbf{x}^{\prime}-\mathbf{x}\right|\right) \\
g^{11}\left(\left|\mathbf{x}^{\prime}-\mathbf{x}\right|\right)
\end{array}\right\} \mathrm{d} V^{\prime}
$$

where

$$
\begin{aligned}
& \left\{\begin{array}{l}
g^{10}\left(\left|\mathbf{x}^{\prime}-\mathbf{x}\right|\right) \\
g^{01}\left(\left|\mathbf{x}^{\prime}-\mathbf{x}\right|\right) \\
g^{20}\left(\left|\mathbf{x}^{\prime}-\mathbf{x}\right|\right) \\
g^{02}\left(\left|\mathbf{x}^{\prime}-\mathbf{x}\right|\right) \\
g^{11}\left(\left|\mathbf{x}^{\prime}-\mathbf{x}\right|\right)
\end{array}\right\}=\left(A^{-1}\left[\begin{array}{lllll}
1 & 0 & 0 & 0 & 0 \\
0 & 1 & 0 & 0 & 0 \\
0 & 0 & 2 & 0 & 0 \\
0 & 0 & 0 & 2 & 0 \\
0 & 0 & 0 & 0 & 1
\end{array}\right]\right)^{T}\left\{\begin{array}{c}
w\left|x^{\prime}-x\right| \\
w\left|y^{\prime}-y\right| \\
w\left|x^{\prime}-x\right|^{2} \\
w\left|y^{\prime}-y\right|^{2} \\
w\left|x^{\prime}-x\right|\left|y^{\prime}-y\right|
\end{array}\right\} \\
& A=\left(\int_{H_{\mathbf{x}}} w\left[\begin{array}{ccccc}
\left|x^{\prime}-x\right|^{2} & \left|x^{\prime}-x\right|\left|y^{\prime}-y\right| & \left|x^{\prime}-x\right|^{3} & \left|x^{\prime}-x\right|\left|y^{\prime}-y\right|^{2} & \left|x^{\prime}-x\right|^{2}\left|y^{\prime}-y\right| \\
\left|x^{\prime}-x\right|\left|y^{\prime}-y\right| & \left|y^{\prime}-y\right|^{2} & \left|x^{\prime}-x\right|^{2}\left|y^{\prime}-y\right| & \left|y^{\prime}-y\right|^{3} & \left|x^{\prime}-x\right|\left|y^{\prime}-y\right|^{2} \\
\left|x^{\prime}-x\right|^{3} & \left|x^{\prime}-x\right|^{2}\left|y^{\prime}-y\right| & \left|x^{\prime}-x\right|^{3} & \left|x^{\prime}-x\right|^{2}\left|y^{\prime}-y\right|^{2} & \left|x^{\prime}-x\right|^{3}\left|y^{\prime}-y\right| \\
\left|x^{\prime}-x\right|\left|y^{\prime}-y\right|^{2} & \left|y^{\prime}-y\right|^{3} & \left|x^{\prime}-x\right|^{2}\left|y^{\prime}-y\right|^{2} & \left|y^{\prime}-y\right|^{4} & \left|x^{\prime}-x\right|\left|y^{\prime}-y\right|^{3} \\
\left|x^{\prime}-x\right|^{2}\left|y^{\prime}-y\right| & \left|x^{\prime}-x\right|\left|y^{\prime}-y\right|^{2} & \left|x^{\prime}-x\right|^{3}\left|y^{\prime}-y\right| & \left|x^{\prime}-x\right|\left|y^{\prime}-y\right|^{3} & \left|x^{\prime}-x\right|^{2}\left|y^{\prime}-y\right|^{2}
\end{array}\right] \mathrm{d} V^{\prime}\right)
\end{aligned}
$$

with $w$ being the weight function defined as [33]

$$
w=e^{-\left(2\left|\mathbf{x}^{\prime}-\mathbf{x}\right| / \delta\right)^{2}}
$$

Besides, according to the derivative differential order, the PDDO is cast into two matrices as

$$
\mathbf{g}_{1}\left(\left|\mathbf{x}^{\prime}-\mathbf{x}\right|\right)=\left[g^{10}\left(\left|\mathbf{x}^{\prime}-\mathbf{x}\right|\right) \quad g^{01}\left(\left|\mathbf{x}^{\prime}-\mathbf{x}\right|\right)\right]
$$

for the first-order derivatives and 


$$
\mathbf{g}_{2}\left(\left|\mathbf{x}^{\prime}-\mathbf{x}\right|\right)=\left[\begin{array}{ll}
g^{20}\left(\left|\mathbf{x}^{\prime}-\mathbf{x}\right|\right) & g^{11}\left(\left|\mathbf{x}^{\prime}-\mathbf{x}\right|\right) \\
g^{11}\left(\left|\mathbf{x}^{\prime}-\mathbf{x}\right|\right) & g^{02}\left(\left|\mathbf{x}^{\prime}-\mathbf{x}\right|\right)
\end{array}\right]
$$

for the second-order derivatives. Consequently, for a vector function, $\mathbf{f}(\mathbf{x})$, the following non-local operator can be obtained as $[34,35]$

$$
\begin{gathered}
\nabla \cdot \mathbf{f}(\mathbf{x})=\int_{H_{\mathbf{x}}} \mathbf{g}_{1}\left(\left|\mathbf{x}^{\prime}-\mathbf{x}\right|\right) \cdot\left(\mathbf{f}\left(\mathbf{x}^{\prime}\right)-\mathbf{f}(\mathbf{x})\right) \mathrm{d} V^{\prime} \\
\nabla \mathbf{f}(\mathbf{x})=\int_{H_{\mathbf{x}}}\left(\mathbf{f}\left(\mathbf{x}^{\prime}\right)-\mathbf{f}(\mathbf{x})\right) \otimes \mathbf{g}_{1}\left(\left|\mathbf{x}^{\prime}-\mathbf{x}\right|\right) \mathrm{d} V^{\prime} \\
\Delta \mathbf{f}(\mathbf{x})=\int_{H_{\mathbf{x}}} \operatorname{tr}\left(\mathbf{g}_{2}\left(\left|\mathbf{x}^{\prime}-\mathbf{x}\right|\right)\right)\left(\mathbf{f}\left(\mathbf{x}^{\prime}\right)-\mathbf{f}(\mathbf{x})\right) \mathrm{d} V^{\prime}
\end{gathered}
$$

\subsection{PDDO fluid model}

In this study, the fluid flow is assumed to be a weakly compressible viscous Newtonian fluid under a laminar flow condition. The Navier-Stokes equations are expressed in PDDO. In the updated Lagrangian description, the current configuration is the reference configuration. Thus, the PDDO is constructed based on the current configuration [34, 35].

The PDDO form of the continuity equation can be presented as [34, 35]

$$
\frac{\partial \rho_{f}\left(\mathbf{y}_{f}, t\right)}{\partial t}=-\rho_{f}\left(\mathbf{y}_{f}, t\right) \int_{H_{\mathbf{x}_{f}}} \mathbf{g}_{1}\left(\left|\mathbf{y}_{f}^{\prime}-\mathbf{y}_{f}\right|\right) \cdot\left(\mathbf{v}_{f}\left(\mathbf{y}_{f}^{\prime}, t\right)-\mathbf{v}_{f}\left(\mathbf{y}_{f}, t\right)\right) \mathrm{d} V_{f}^{\prime}
$$

The PDDO form of the momentum equation can be presented as [34, 35]

$$
\rho_{f}\left(\mathbf{y}_{f}, t\right) \ddot{\mathbf{u}}_{f}\left(\mathbf{y}_{f}, t\right)=\int_{H_{\mathbf{x}_{f}}}\left[\begin{array}{l}
-\left(P_{f}\left(\mathbf{y}_{f}^{\prime}, t\right)-P_{f}\left(\mathbf{y}_{f}, t\right)\right) \mathbf{g}_{1}\left(\left|\mathbf{y}_{f}^{\prime}-\mathbf{y}_{f}\right|\right) \\
+\mu_{f}\left(\mathbf{v}_{f}\left(\mathbf{y}_{f}^{\prime}, t\right)-\mathbf{v}_{f}\left(\mathbf{y}_{f}, t\right)\right) \operatorname{Tr}\left(\mathbf{g}_{2}\left(\left|\mathbf{y}_{f}^{\prime}-\mathbf{y}_{f}\right|\right)\right)
\end{array}\right] \mathrm{d} V_{f}^{\prime}+\mathbf{b}_{f}\left(\mathbf{y}_{f}, t\right)
$$

where $\ddot{\mathbf{u}}_{f}$ represents the acceleration and $P_{f}$ represents the pressure of the fluid. The parameters $\mathbf{y}_{f}$ and $\mathbf{y}_{f}^{\prime}$ are the updated positions of the fluid particles, as shown in Fig. 2.

The equation of state is expressed as [56] 


$$
P_{f}=\frac{\rho_{f, 0} c_{f}^{2}}{\gamma_{f}}\left(\left(\frac{\rho_{f}}{\rho_{f, 0}}\right)^{\gamma_{f}}-1\right)
$$

where $\rho_{f, 0}$ is the initial fluid density, $\gamma_{f}$ is the material constant ranging from 1 to 7 [56]. The term $c_{f}$ is the artificial speed of sound for fluid, which is assumed to be 10 times larger than the maximum fluid velocity magnitude $\left|\mathbf{v}_{f, \max }\right|[57]$.

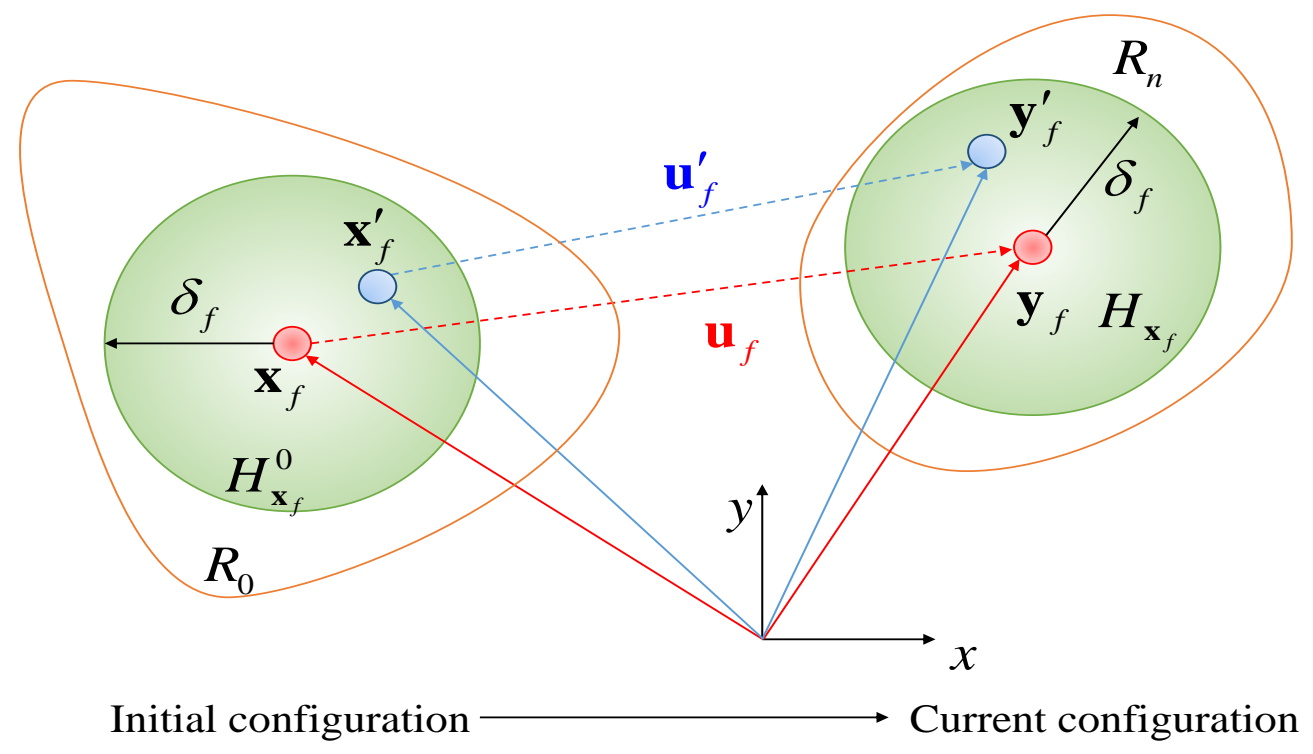

Fig. 2 PDDO kinetic quantities in updated Lagrangian description

\section{Numerical implementations for fluid-structure simulation}

The algorithm of the interaction implementation between the fluid and elastic structure is a key issue in FSI problems. As illustrated in Fig. 3, two different sets of PD particles are used to represent the structure and the fluid, i.e. green particles for structure and blue particles for fluid. For the particles near the fluid-structure interface, $\Gamma$, there are four kinds of PD interactions between them: structure-structure interaction (green colour), fluid-fluid interaction (blue colour), structure (central)-fluid (family member) interaction (red colour), and fluid (central)-structure (family member) interaction (yellow colour).

For the first two kinds of interactions (the single-phase interactions, the green one and blue one), the OSB-PD model and PDDO model can be utilized to simulate the solid and fluid, 
which are presented in Section 4.1. The numerical implementation for the other two kinds of PD interactions (the red one and the yellow one) is presented in Section 4.3.

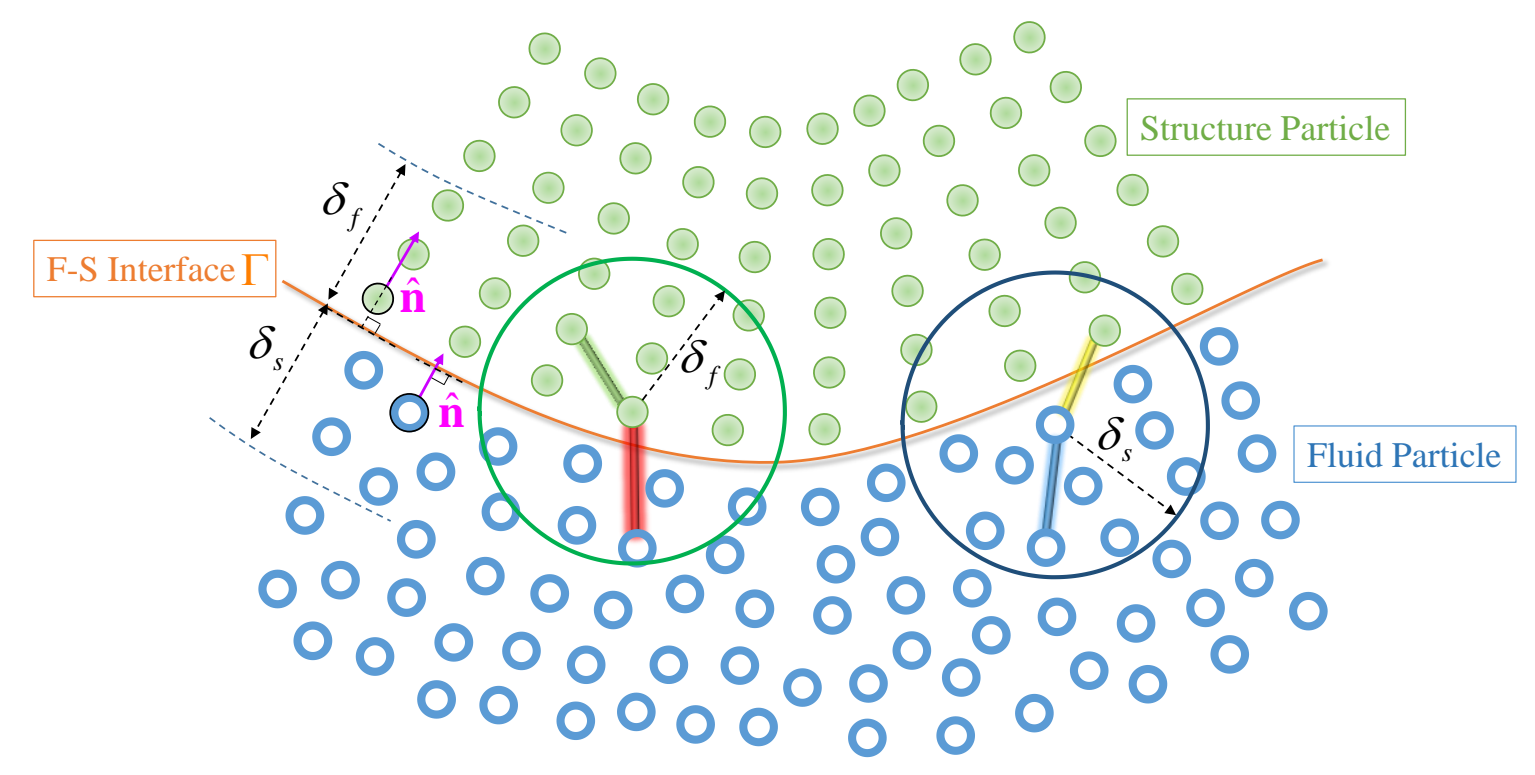

Fig. 3 Fluid-Structure interface illustration

\subsection{Discretised form of PD governing equations}

The governing equations are expressed by using the discretized forms. Since the OSB-PD theory in the total Lagrangian description is used for the structural model, the solid particle is denoted by its initial position, $\mathbf{x}_{s}$. The fluid flow model is in the updated Lagrangian description. Thus, the fluid particle is denoted by its position in the current configuration, $\mathbf{y}_{s}$. Correspondingly, the peridynamic differential operators associated with two PD particles are functions of their relative current positions.

Equation of motion for mechanical deformations:

$$
\ddot{\mathbf{u}}_{s, i}^{n+1}=\frac{1}{\rho_{s}} \sum_{j=1}^{N_{s, i}}\left(\frac{2 \delta_{s} a d \Lambda_{i j}}{\left|\mathbf{x}_{s, j}-\mathbf{x}_{s, i}\right|}\left(\theta_{s, i}+\theta_{s, j}\right)+4 \delta_{s} b s_{i j}\right) \frac{\mathbf{y}_{s, j}-\mathbf{y}_{s, i}}{\left|\mathbf{y}_{s, j}-\mathbf{y}_{s, i}\right|} V_{s, j}+\frac{1}{\rho_{s}} \mathbf{b}_{s, i}^{n+1}
$$

with

$$
\theta_{s, i}=\sum_{j=1}^{N_{s, i}}\left(d \delta_{s} s_{i j} \Lambda_{i j}\right) V_{s, j}, \theta_{s, j}=\sum_{i=1}^{N_{s, j}}\left(d \delta_{s} s_{i j} \Lambda_{i j}\right) V_{s, i}
$$




$$
\begin{gathered}
s_{i j}=\frac{\left|\mathbf{y}_{s, j}-\mathbf{y}_{s, i}\right|-\left|\mathbf{x}_{s, j}-\mathbf{x}_{s, i}\right|}{\left|\mathbf{x}_{s, j}-\mathbf{x}_{s, i}\right|} \\
\Lambda_{i j}=\left(\frac{\mathbf{y}_{s, j}-\mathbf{y}_{s, i}}{\left|\mathbf{y}_{s, j}-\mathbf{y}_{s, i}\right|}\right) \cdot\left(\frac{\mathbf{x}_{s, j}-\mathbf{x}_{s, i}}{\left|\mathbf{x}_{s, j}-\mathbf{x}_{s, i}\right|}\right)
\end{gathered}
$$

where $\ddot{\mathbf{u}}_{s, i}^{n+1}$ is the acceleration of solid particle $i$ at time step $n+1 . N_{s, i}$ is the total number of the family members belonging to solid of solid particle $i . \mathbf{x}_{s, i}$ and $\mathbf{x}_{s, j}$ are the initial positions of solid particle $i$ and $j$. Correspondingly, $\mathbf{y}_{s, i}$ and $\mathbf{y}_{s, j}$ are the current positions of particle $i$ and $j . \theta_{s, i}$ and $\theta_{s, j}$ are the dilatations of particle $i$ and $j . s_{i j}$ is the bond stretch between particle $i$ and $j . V_{s, j}$ is the volume of the solid particle $j$. The subscript $\left({ }_{s}\right)$ denotes the solid particles. The superscript $\left({ }^{n+1}\right)$ denotes the updated configuration.

Navier-Stokes equations for fluid:

$$
\begin{aligned}
\rho_{f, i}^{n+1} & =\rho_{f, i}^{n}-\rho_{f, i}^{n} \Delta t \sum_{j=1}^{N_{f, i}}\left[\left(\mathbf{v}_{f, j}^{n}-\mathbf{v}_{f, i}^{n}\right) \cdot \mathbf{g}_{1}\left(\left|\mathbf{y}_{f, j}^{n}-\mathbf{y}_{f, i}^{n}\right|\right)\right] V_{f, j}^{n} \\
\ddot{\mathbf{u}}_{f, i}^{n+1}= & \frac{1}{\rho_{f, i}^{n+1}}\left(\mu_{f} \sum_{j=1}^{N_{f, i}}\left(\mathbf{v}_{f, j}^{n}-\mathbf{v}_{f, i}^{n}\right) \operatorname{Tr}\left(\mathbf{g}_{2}\left(\left|\mathbf{y}_{f, j}^{n}-\mathbf{y}_{f, i}^{n}\right|\right)\right) V_{f, j}^{n}\right) \\
& -\frac{1}{\rho_{f, i}^{n+1}}\left(\sum_{j=1}^{N_{f, i}}\left(P_{f, j}^{n}-P_{f, i}^{n}\right) \mathbf{g}_{1}\left(\left|\mathbf{y}_{f, j}^{n}-\mathbf{y}_{f, i}^{n}\right|\right) V_{f, j}^{n}\right)+\frac{1}{\rho_{f, i}^{n+1}} \mathbf{b}_{f, i}^{n+1}
\end{aligned}
$$

The subscript $\left({ }_{f}\right)$ denotes that the particles belong to fluids. $\rho_{f, i}^{n+1}$ and $\ddot{\mathbf{u}}_{f, i}^{n+1}$ represent the density and acceleration of fluid particle $i$ in the updated configuration $t=t_{n+1} . N_{f, i}$ is the total number of the family members belonging to the fluid system of particle $i . V_{f, j}^{n}$ is the volume of fluid particle $j$ in the current configuration.

The velocity, displacement, and position for solid and fluid particles are updated by the velocity Verlet algorithm (2 $2^{\text {nd }}$ order) [58] as

$$
\mathbf{v}_{s, i}^{n+1}=\mathbf{v}_{s, i}^{n}+\frac{1}{2}\left(\ddot{\mathbf{u}}_{s, i}^{n+1}+\ddot{\mathbf{u}}_{s, i}^{n}\right) \Delta t, \mathbf{v}_{f, i}^{n+1}=\mathbf{v}_{f, i}^{n}+\frac{1}{2}\left(\ddot{\mathbf{u}}_{f, i}^{n+1}+\ddot{\mathbf{u}}_{f, i}^{n}\right) \Delta t
$$




$$
\begin{gathered}
\mathbf{u}_{s, i}^{n+1}=\mathbf{u}_{s, i}^{n}+\mathbf{v}_{s, i}^{n+1} \Delta t+\frac{1}{2} \ddot{\mathbf{u}}_{s, i}^{n} \Delta t, \mathbf{u}_{f, i}^{n+1}=\mathbf{u}_{f, i}^{n}+\mathbf{v}_{f, i}^{n} \Delta t+\frac{1}{2} \ddot{\mathbf{u}}_{f, i}^{n} \Delta t^{2} \\
\mathbf{y}_{s, i}^{n+1}=\mathbf{x}_{s, i}+\mathbf{u}_{s, i}^{n+1}, \mathbf{y}_{f, i}^{n+1}=\mathbf{x}_{f, i}+\mathbf{u}_{f, i}^{n+1}
\end{gathered}
$$

In the present study, a uniform initial node spacing is adopted for both fluid and solid. Hence the horizon size for fluid $\delta_{f}$ and the horizon size for solid $\delta_{s}$ equal to each other, i.e. $\delta_{f}=\delta_{s}=\delta$

\subsection{Numerical treatment for the fluid model}

\subsubsection{Free surface detection}

Similar to MPS scheme, number density is calculated for detecting the free surface as proposed by Koshizuka et al. [59]. The number density can reflect the number of family members where a larger one represents more family members and a smaller one represents fewer family members. The initial and current number densities can reflect the level of the number of family members in the initial and current configuration, respectively. The initial and current number densities of particle $i$ are calculated as [59]

$$
\begin{aligned}
& d^{(0)}(i)=\sum_{j=1}^{N_{i}}\left(e^{-\left(\left|\mathbf{x}_{j}-\mathbf{x}_{i}\right| / h\right)^{2}}-e^{-9}\right) /\left(\pi h^{2}\left(1-10 e^{-9}\right)\right) \\
& d^{(n)}(i)=\sum_{j=1}^{N_{i}}\left(e^{-\left(\left|\mathbf{y}_{j}-\mathbf{y}_{i}\right| / h\right)^{2}}-e^{-9}\right) /\left(\pi h^{2}\left(1-10 e^{-9}\right)\right)
\end{aligned}
$$

where $h$ is the smooth length defined as $h=1.2 \Delta x$ [60]. Particle $i$ will become a free-surface fluid particle, and its pressure will be forced as $P_{i}=0$, if the following criterion is satisfied [59]

$$
d^{(n)}(i)<0.9 d^{(0)}(i)
$$

\subsubsection{Initial damping}

The initial damping technique is utilized to make the PDDO fluid model more stable at the initial stage. Initial damping time, $t_{\text {damp }}$, is chosen during which the acceleration of each PD fluid particle is multiplied by a factor, $f_{\text {damp }}(t)$ [61]

$$
\left(\ddot{\mathbf{u}}_{f, i}^{n}\right)^{\text {damped }}=\ddot{\mathbf{u}}_{f, i}^{n} f_{\text {damp }}(t)
$$




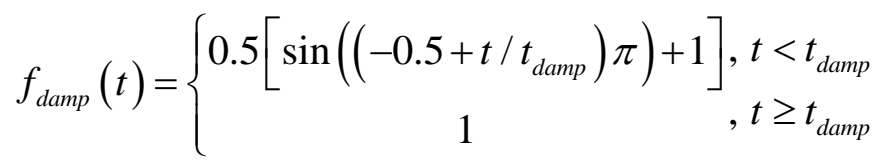

where $\left(\ddot{\mathbf{u}}_{f, i}^{n}\right)^{\text {damped }}$ is the acceleration of fluid particle $i$ after damping.

\subsubsection{Moving least-square scheme for smoothing}

The moving least squares (MLS) method [62] is adopted for every $n_{M L S}$ time step to smooth the fluid velocity and density profiles as

$$
\begin{gathered}
\mathbf{v}_{f}^{\text {smoothed }}\left(\mathbf{y}_{f, i}, t\right)=\frac{\sum_{j} \mathbf{v}_{f}\left(\mathbf{y}_{f, j}, t\right) w_{M L S}\left(\mathbf{y}_{f, i}, \mathbf{y}_{f, j}\right)}{\sum_{j} w_{M L S}\left(\mathbf{y}_{f, i}, \mathbf{y}_{f, j}\right)} \\
\rho_{f}^{\text {smoothed }}\left(\mathbf{y}_{f, i}, t\right)=\sum_{j} m_{f, j} w_{M L S}\left(\mathbf{y}_{f, i}, \mathbf{y}_{f, j}\right)
\end{gathered}
$$

where the smoothing weight function can be expressed as [62]

$$
\begin{aligned}
& w_{M L S}\left(\mathbf{y}_{f, i}, \mathbf{y}_{f, j}\right)=\left[\beta_{0}\left(\left|\mathbf{y}_{f, i}-\mathbf{y}_{f, j}\right|\right)+\beta_{1}\left(\left|\mathbf{y}_{f, i}-\mathbf{y}_{f, j}\right|\right)\left(-\varsigma_{x}\right)\right] w_{0}\left(\left|\mathbf{y}_{f, j}-\mathbf{y}_{f, i}\right|\right) \\
& +\beta_{2}\left(\left|\mathbf{y}_{f, i}-\mathbf{y}_{f, j}\right|\right)\left(-\varsigma_{y}\right) w_{0}\left(\left|\mathbf{y}_{f, j}-\mathbf{y}_{f, i}\right|\right) \\
& \boldsymbol{\beta}\left(\left|\mathbf{y}_{f, i}-\mathbf{y}_{f, j}\right|\right)=\left(\begin{array}{l}
\beta_{0} \\
\beta_{1} \\
\beta_{2}
\end{array}\right)=\mathbf{S}^{-1}\left(\left|\mathbf{y}_{f, i}-\mathbf{y}_{f, j}\right|\right)\left[\begin{array}{l}
1 \\
0 \\
0
\end{array}\right] \\
& \mathbf{S}\left(\left|\mathbf{y}_{f, i}-\mathbf{y}_{f, j}\right|\right)=\sum_{j=1}^{N_{i}} w_{0}\left(\left|\mathbf{y}_{f, i}-\mathbf{y}_{f, j}\right|\right)\left[\begin{array}{ccc}
1 & -\varsigma_{x} & -\varsigma_{y} \\
-\varsigma_{x} & \left(\varsigma_{x}\right)^{2} & \varsigma_{x} \varsigma_{y} \\
-\varsigma_{y} & \varsigma_{x} \varsigma_{y} & \left(\varsigma_{y}\right)^{2}
\end{array}\right] \\
& \mathbf{y}_{f, j}-\mathbf{y}_{f, i}=\left[\begin{array}{l}
\varsigma_{x} \\
\varsigma_{y}
\end{array}\right] \\
& w_{0}\left(\left|\mathbf{y}_{f, i}-\mathbf{y}_{f, j}\right|\right)=\left(e^{-\left(\left(\left|\mathbf{y}_{f, i}-\mathbf{y}_{f, j}\right|\right) / h\right)^{2}}-e^{-9}\right) /\left(\pi h^{2}\left(1-10 e^{-9}\right)\right),\left(\left|\mathbf{y}_{f, i}-\mathbf{y}_{f, j}\right|\right)<\delta
\end{aligned}
$$

where $h$ is the smooth length defined as $h=1.2 \Delta x[60]$ 


\subsubsection{Relative deformation gradient in PDDO form}

In the numerical simulation, the updated Lagrangian description is adopted for fluid, as explained in [34]. Therefore, the reference configuration is referred to as the current configuration. The PDDO is reconstructed every time step based on the updated family member array. Furthermore, the relative deformation gradient is calculated to estimate the fluid particle volume in the updated configuration.

As provided in Appendix A, the PD form of deformation gradient [63] is modified for a fluid particle at current and updated configurations as

$$
\begin{aligned}
& \mathbf{F}_{n}\left(\mathbf{y}_{f, i}^{n}\right)=\frac{2}{\pi h_{\text {thick }} \delta_{f}^{4}} \sum_{j=1}^{N_{i}}\left(\frac{\delta_{f}}{\left|\mathbf{y}_{f, j}^{n}-\mathbf{y}_{f, i}^{n}\right|}\right)^{2}\left(\mathbf{y}_{f, j}^{n}-\mathbf{y}_{f, i}^{n}\right) \otimes\left(\mathbf{y}_{f, j}^{n}-\mathbf{y}_{f, i}^{n}\right) V_{f, j}^{n} \\
& \mathbf{F}_{n+1}\left(\mathbf{y}_{f, i}^{n}\right)=\frac{2}{\pi h_{t h i c k} \delta_{f}^{4}} \sum_{j=1}^{N_{i}}\left(\frac{\delta_{f}}{\left|\mathbf{y}_{f, j}^{n}-\mathbf{y}_{f, i}^{n}\right|}\right)^{2}\left(\mathbf{y}_{f, j}^{n+1}-\mathbf{y}_{f, i}^{n+1}\right) \otimes\left(\mathbf{y}_{f, j}^{n}-\mathbf{y}_{f, i}^{n}\right) V_{f, j}^{n}
\end{aligned}
$$

Consequently, the PDDO form of relative deformation gradient can be defined as [43, 63]

$$
\begin{aligned}
& \Delta \mathbf{F}\left(\mathbf{y}_{f, i}^{n}\right)=\frac{\mathbf{F}_{n+1}\left(\mathbf{y}_{f, i}^{n}\right)}{\mathbf{F}_{n}\left(\mathbf{y}_{f, i}^{n}\right)} \\
& =\frac{\left[\sum_{j=1}^{N_{i}}\left(\frac{\delta_{f}}{\left|\mathbf{y}_{f, j}^{n}-\mathbf{y}_{f, i}^{n}\right|}\right)^{2}\left(\mathbf{y}_{f, j}^{n+1}-\mathbf{y}_{f, i}^{n+1}\right) \otimes\left(\mathbf{y}_{f, j}^{n}-\mathbf{y}_{f, i}^{n}\right) V_{f, j}^{n}\right]}{\left[\sum_{j=1}^{N_{i}}\left(\frac{\delta_{f}}{\left|\mathbf{y}_{f, j}^{n}-\mathbf{y}_{f, i}^{n}\right|}\right)^{2}\left(\mathbf{y}_{f, j}^{n}-\mathbf{y}_{f, i}^{n}\right) \otimes\left(\mathbf{y}_{f, j}^{n}-\mathbf{y}_{f, i}^{n}\right) V_{f, j}^{n}\right]}
\end{aligned}
$$

Finally, the updated volume of a fluid point is approximated as

$$
V_{f}^{n+1}=\operatorname{det}(\Delta \mathrm{F}) V_{f}^{n}
$$

Then Eq.(31) is substituted into Eqs.(18) and (19) to represent the volume of fluid particle $j$.

\subsubsection{Artificial viscosity in PDDO form}

The artificial viscosity, $\Pi$ which is usually utilized in mesh-free methods, e.g. SPH method [46, 64], can be adopted in the current PDDO fluid model. Consequently, the momentum equation becomes 


$$
\begin{aligned}
\rho_{f, i}^{n+1} \mathbf{\mathbf { u }}_{f, i}^{n+1} & =\left(\mu_{f} \sum_{j=1}^{N_{f, i}}\left(\mathbf{v}_{f, j}^{n}-\mathbf{v}_{f, i}^{n}\right) \operatorname{Tr}\left(\mathbf{g}_{2}\left(\left|\mathbf{y}_{f, j}^{n}-\mathbf{y}_{f, i}^{n}\right|\right)\right) V_{f, j}^{n}\right) \\
& -\left(\sum_{j=1}^{N_{f, i}}\left(P_{f, j}^{n}-P_{f, i}^{n}\right) \mathbf{g}_{1}\left(\left|\mathbf{y}_{f, j}^{n}-\mathbf{y}_{f, i}^{n}\right|\right) V_{f, j}^{n}\right)+\mathbf{b}_{f, i}^{n+1}+\nabla \prod_{i}
\end{aligned}
$$

with

$$
\nabla \prod_{i}=\left\{\begin{array}{l}
\sum_{j=1}^{N_{f, i}} \frac{\alpha h c_{f}\left(\left(\mathbf{v}_{f, j}^{n}-\mathbf{v}_{f, i}^{n}\right) \cdot\left(\mathbf{y}_{f, j}^{n}-\mathbf{y}_{f, i}^{n}\right)\right)}{\left|\mathbf{y}_{f, j}^{n}-\mathbf{y}_{f, i}^{n}\right|^{2}+(0.1 h)^{2}} \mathbf{g}_{1}\left(\left|\mathbf{y}_{f, j}^{n}-\mathbf{y}_{f, i}^{n}\right|\right) V_{f, j}^{n}, \\
\text { if }\left(\mathbf{v}_{f, j}^{n}-\mathbf{v}_{f, i}^{n}\right) \cdot\left(\mathbf{y}_{f, j}^{n}-\mathbf{y}_{f, i}^{n}\right)<0 \\
0, \text { otherwise }
\end{array}\right.
$$

where $h=1.2 \Delta x$ [39] and the constant parameter $\alpha$ is set as $\alpha=0.02$ [46] in the current study. The term $c_{f}$ is the artificial speed of sound provided in Eq.(16). Therefore, in the present study, both the physical viscosity and artificial viscosity are considered in the momentum equation.

\subsubsection{Particle shifting technique for preventing particle clustering}

If the free surface detecting approach provided in section 4.2.1 is applied, the internal fluid particles may be mistakenly treated as free surface particles [65], leading to an over prediction of the number of free surface particles. As a result, the particle clustering on the free surface will become more obvious as the time increases. Therefore, the particle shifting technique (PST) [66] is utilized in the present model. The position of fluid particle $i$ in the updated configuration is shifted by a shifting distance $\left(\delta \mathbf{u}_{f, i}^{n+1}\right)^{P S T}$ as [66]

$$
\left(\mathbf{y}_{f, i}^{n+1}\right)^{\text {corrected }}=\mathbf{x}_{f, i}+\left(\mathbf{u}_{f, i}^{n+1}\right)^{\text {shifted }}
$$

and

$$
\left(\mathbf{u}_{f, i}^{n+1}\right)^{\text {shifted }}=\mathbf{u}_{f, i}^{n+1}+\left(\delta \mathbf{u}_{f, i}^{n+1}\right)^{P S T}
$$

where $\left(\delta \mathbf{u}_{f, i}^{n+1}\right)^{P S T}$ is calculated as [66] 


$$
\left(\delta \mathbf{u}_{f, i}\right)^{P S T}=C_{P S T}\left|\mathbf{v}_{f, \max }\right| \Delta t\left(\sum_{j=1}^{N_{f i}} \frac{\left|\frac{1}{N_{f, i}} \sum_{j=1}^{N_{f i}}\right| \mathbf{y}_{f, j}^{n}-\left.\mathbf{y}_{f, i}^{n}\right|^{2}}{\left|\mathbf{y}_{f, j}^{n}-\mathbf{y}_{f, i}^{n}\right|^{2}} \frac{\mathbf{y}_{f, j}^{n}-\mathbf{y}_{f, i}^{n}}{\left|\mathbf{y}_{f, j}^{n}-\mathbf{y}_{f, i}^{n}\right|}\right)
$$

The constant parameter is chosen as $C_{P S T}=0.02$ in the current study [66]. Besides, an upper limit, $0.2 \Delta x$, is given to the shifting distance $\left(\delta \mathbf{u}_{f, i}^{n+1}\right)^{P S T}$ to avoid excessive diffusion [66].

\subsubsection{Fluid-structure interface collision model}

The fluid-structure interface collision model is applied for preventing material points to enter the solid structure [67]. The solid wall is simulated as a reflective boundary condition.

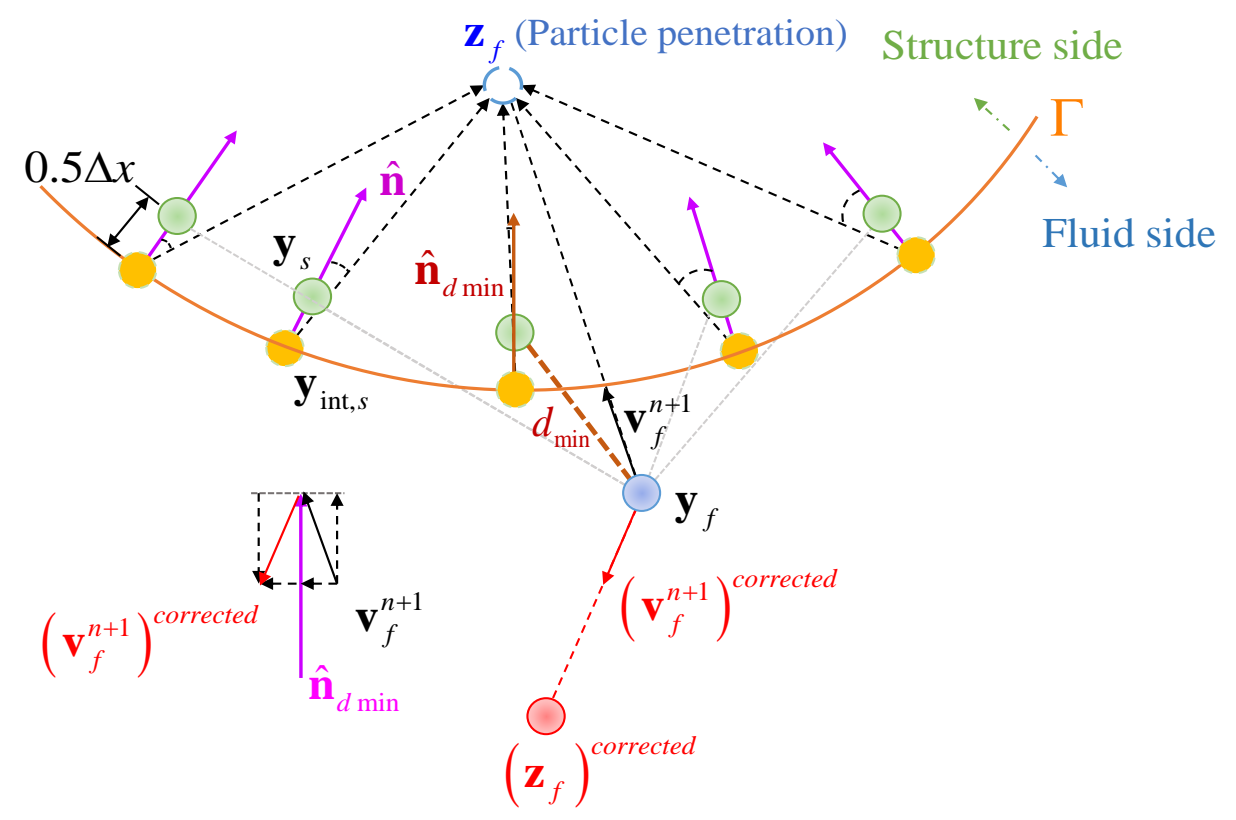

Fig. 4 Collision model for fluid-structure interface condition

In Fig. 4, the fluid particle at $\mathbf{y}_{f}$ is located within the fluid-structure interface region (defined in Fig. 3). The solid particles within the interface-nearest layer (these solid particles have the shortest distances to the interface) are considered here as the family members of fluid particle at $\mathbf{y}_{f}$, e.g. the solid particle at $\mathbf{y}_{s}$ belongs to the family members of the fluid particle at $\mathbf{y}_{f}$. 
A fictitious particle at $\mathbf{y}_{\mathrm{int}, s}=\mathbf{y}_{s}-0.5 \Delta x \hat{\mathbf{n}}\left(\mathbf{y}_{s}\right)$ is defined as the corresponding interface particle of the solid particle at $\mathbf{y}_{s}$, shown as the yellow circle in Fig. 4. The position $\mathbf{y}_{\mathrm{int}, s}$ of this fictitious particle is assumed to be on the fluid-structure interface, as shown in Fig. 4.

Then the collision model is applied in two steps:

Step 1) The first step is to check if the fluid particle at $\mathbf{y}_{f}$ penetrates the interface $\Gamma$.

The new position of the fluid particle at $\mathbf{y}_{f}$ is updated according to Eq.(22). The new location of the fluid particle is denoted as $\mathbf{z}_{f}$. Then for all the solid family member particles considered in the collision model, e.g. solid particle at $\mathbf{y}_{s}$, the following criterion is checked

$$
\hat{\mathbf{n}}\left(\mathbf{y}_{s}\right) \cdot\left(\mathbf{z}_{f}-\mathbf{y}_{\mathrm{int}, s}\right)>0
$$

If the criterion in Eq.(37) is satisfied, it is concluded that the particle at $\mathbf{z}_{f}$ penetrates the solid (shown as dashed blue circle in Fig. 4).

Step 2) If the fluid particle penetrates the structure, its velocity should be corrected accordingly.

First, find the solid family member with the shortest relative distance to the fluid particle at $\mathbf{y}_{f}$ and its unit normal as

$$
\begin{gathered}
d_{\min }=\min \left(\left|\mathbf{y}_{s}-\mathbf{y}_{f}\right|\right), \mathbf{y}_{s} \in H_{\mathbf{y}_{f}}^{n+1} \\
\hat{\mathbf{n}}_{d \text { min }}=\hat{\mathbf{n}}\left(\mathbf{y}_{s, d \text { min }}\right)
\end{gathered}
$$

Subsequently, the velocity of the fluid particle at $\mathbf{y}_{f}$ is corrected as

$$
\left(\mathbf{v}_{f}^{n+1}\right)^{\text {corrected }}=\mathbf{v}_{f}^{n+1}-2\left(\mathbf{v}_{f}^{n+1} \cdot \hat{\mathbf{n}}_{d \text { min }}\right) \hat{\mathbf{n}}_{d \min }, \text { if } \mathbf{v}_{f}^{n+1} \cdot \hat{\mathbf{n}}_{d \min }>0
$$

Here $\mathbf{v}_{f}^{n+1}$ is the velocity predicted by Eq.(20). The updated corrected displacement and position become

$$
\left(\mathbf{u}_{f, i}^{n+1}\right)^{\text {corrected }}=\mathbf{u}_{f, i}^{n}+\left(\mathbf{v}_{f}^{n+1}\right)^{\text {corrected }} \Delta t+\frac{1}{2} \ddot{\mathbf{u}}_{f}^{n} \Delta t^{2}
$$




$$
\left(\mathbf{z}_{f}\right)^{\text {corrected }}=\mathbf{x}_{f}+\left(\mathbf{u}_{f}^{n+1}\right)^{\text {corrected }}
$$

\subsection{Boundary and FSI treatment}

For flow simulation, the elastic structure serves as a moving boundary condition with the deformed configuration. The solid particle at $\mathbf{y}_{s}$ is treated as the boundary particle of fluid $\mathbf{y}_{f, \text { boundary }}$ which occupies the same position as $\mathbf{y}_{s}$.

On the other hand, the fluid effect on the structural deformation is considered via the fluid pressure acting as an external force [4]. The details are provided in the following content in this section.

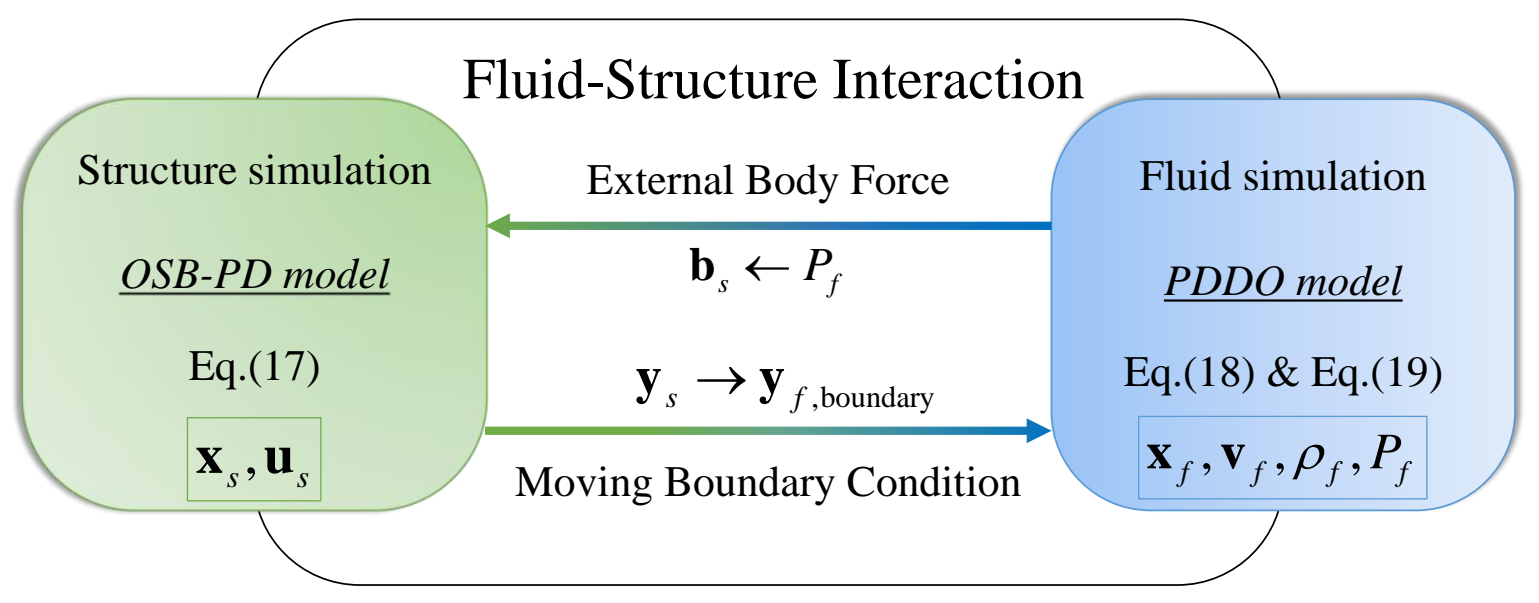

Fig. 5 FSI methodology scheme

\subsubsection{Interface definition and its normal}

In dealing with the interaction between the elastic structure and fluid particles, the definition of the interface domain and its normal is one of the key issues. Since PD is a nonlocal theory, the interface region between the fluid and the elastic structure has a fixed thickness, being twice of the horizon size $2 \delta$, as shown in Fig. 3. Because of the movement of the structure and fluid particles, the interface, as well as its normal direction $\hat{\mathbf{n}}$ (see Fig. 3), changes with time. The colour function method which is adopted in the volume of fraction (VOF) [68] method is employed in the present study. The structure and the fluid particles are identified by a predefined colour function as

$$
c\left(\mathbf{x}_{i}\right)=\left\{\begin{array}{l}
1, \text { for particle } \mathbf{x} \text { belonging to fluid, } \mathbf{x}_{i}=\mathbf{x}_{f} \\
2, \text { for particle } \mathbf{x} \text { belonging to solid, } \mathbf{x}_{i}=\mathbf{x}_{s}
\end{array}\right.
$$


The colour function indicates that a particle belongs to the fluid or the structure, which will not change during the simulation process. Hence, the colour function in Eq.(42) is expressed based on the initial configuration. Then the gradient of the colour function at point $\mathbf{x}_{i}$ can be obtained via PDDO approximation as

$$
\mathbf{c}_{g}\left(\mathbf{x}_{i}\right)=\nabla c\left(\mathbf{x}_{i}\right)=\sum_{j=1}^{N_{i}}\left(c\left(\mathbf{x}_{j}\right)-c\left(\mathbf{x}_{i}\right)\right) \mathbf{g}_{1}\left(\left|\mathbf{y}_{j}-\mathbf{y}_{i}\right|\right) V_{j}
$$

It should be noted that particle $\mathbf{x}_{i}$ and $\mathbf{x}_{j}$ in Eq.(43) can be both solid particles, or both fluid particles, or one fluid particle and one solid particle. Besides, it is obvious that if the particle $\mathbf{x}_{i}$ is not in the interface region, the value of $\nabla c\left(\mathbf{x}_{i}\right)$ is zero. Accordingly, the normal direction vector of the interface can be approximated as

$$
\mathbf{n}\left(\mathbf{x}_{i}\right)=\mathbf{c}_{g}\left(\mathbf{x}_{i}\right)=\sum_{j=1}^{N_{i}}\left(c\left(\mathbf{x}_{j}\right)-c\left(\mathbf{x}_{i}\right)\right) \mathbf{g}_{1}\left(\left|\mathbf{y}_{j}-\mathbf{y}_{i}\right|\right) V_{j}
$$

It can be inferred from Eq.(44) that if the neighbourhood of point $\mathbf{x}_{i}$ is fully located within the single-phase region, i.e. either the structure domain or the fluid domain, the value of the normal direction becomes zero. On the contrary, if the particle is in the interface region, the magnitude of its normal direction will not be zero. Then, the unit normal direction vector is calculated as

$$
\hat{\mathbf{n}}\left(\mathbf{x}_{i}\right)=\frac{\mathbf{c}_{g}\left(\mathbf{x}_{i}\right)}{\left|\mathbf{c}_{g}\left(\mathbf{x}_{i}\right)\right|}
$$

In the present study, a cut-off value is set as $\varepsilon=1.0 \times 10^{-2} / \Delta x$ [69] for the normal vector, smaller than which the unit normal becomes zero, i.e.

$$
\mathbb{N}\left(\mathbf{x}_{i}\right)=\left\{\begin{array}{l}
1, \text { if }\left|\mathbf{c}_{g}\left(\mathbf{x}_{i}\right)\right|>\varepsilon \\
0, \text { otherwise }
\end{array}\right.
$$

and 


$$
\hat{\mathbf{n}}\left(\mathbf{x}_{i}\right)=\left\{\begin{array}{l}
\frac{\mathbf{c}_{g}\left(\mathbf{x}_{i}\right)}{\left|\mathbf{c}_{g}\left(\mathbf{x}_{i}\right)\right|}=\frac{\sum_{j=1}^{N_{i}}\left(c\left(\mathbf{x}_{j}\right)-c\left(\mathbf{x}_{i}\right)\right) \mathbf{g}_{1}\left(\left|\mathbf{y}_{j}-\mathbf{y}_{i}\right|\right) V_{j}}{\left|\sum_{j=1}^{N_{i}}\left(c\left(\mathbf{x}_{j}\right)-c\left(\mathbf{x}_{i}\right)\right) \mathbf{g}_{1}\left(\left|\mathbf{y}_{j}-\mathbf{y}_{i}\right|\right) V_{j}\right|}, \text { if } \mathbb{N}\left(\mathbf{x}_{i}\right)=1 \\
0, \text { if } \mathbb{N}\left(\mathbf{x}_{i}\right)=0
\end{array}\right.
$$

It can be observed that since the term $\left(c\left(\mathbf{x}_{j}\right)-c\left(\mathbf{x}_{i}\right)\right)$ can be positive or negative, the unit normal direction always points from the fluid phase to the structure phase.

\subsubsection{Effect of fluid flow on structure}

The fluid effect on the structure deformation is simulated in terms of volumetric external body force. The similar approach developed by Antoci et al. [4] is adopted.

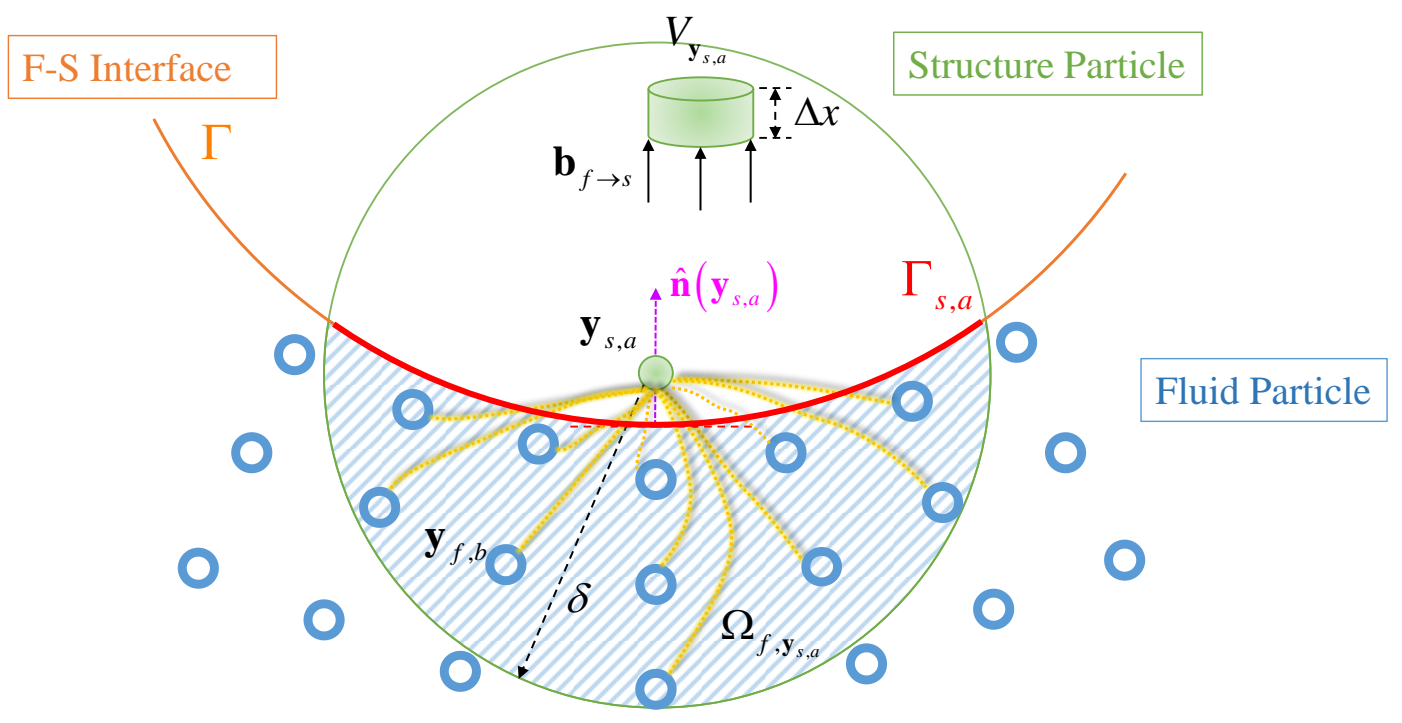

Fig. 6 PD Interactions of the interface structure particle at $\mathbf{y}_{s, a}$

The fluid effect acting on solid particle at $\mathbf{y}_{s, a}$ can be approximated by the pressure of the fluid particles as

$$
P_{s, a}=\frac{\sum_{b \in \Omega_{f, y s, a}} \frac{m_{f, b}}{\rho_{f, b}} P_{f, b} w_{0}\left(\left|\mathbf{y}_{f, b}-\mathbf{y}_{s, a}\right|\right)}{\sum_{b \in \Omega_{f, y_{s, a}}} \frac{m_{f, b}}{\rho_{f, b}} w_{0}\left(\left|\mathbf{y}_{f, b}-\mathbf{y}_{s, a}\right|\right)}
$$


where $\Omega_{f, \mathbf{y}_{s, a}}$ represents the interaction domain of solid particle at $\mathbf{y}_{s, a}$ within the fluid domain as shown in Fig. 6, and $w_{0}$ is the weighted function as defined in Eq. (28e). $P_{f, b}$ is the pressure, $m_{f, b}$ is the mass, and $\rho_{f, b}$ is the density of fluid particle at $\mathbf{y}_{f, b}$. Note that particle at $\mathbf{y}_{f, b}$ belongs to the family members of the point at $\mathbf{y}_{s, a}$ as demonstrated in Fig. 6 . The mass of the fluid particle, $m_{f, b}$, is calculated by using the initial values of density and volume. It's assumed constant during the time integration as

$$
m_{f, b}=\left(\rho_{f, b} V_{f, b}\right)_{t=0}
$$

As explained by Antoci et al. [4], Eq.(48) can also be simplified as

$$
P_{s, a}=2 \sum_{b \in \Omega_{f, y_{s, a}}} \frac{m_{f, b}}{\rho_{f, b}} P_{f, b} w_{0}\left(\left|\mathbf{y}_{f, b}-\mathbf{y}_{s, a}\right|\right)
$$

which provides a better and more stable solution.

The volumetric force exerted from the fluid particles to the structure particle at $\mathbf{y}_{s, a}$ can be approximated as

$$
\mathbf{b}_{f \rightarrow s}\left(\mathbf{y}_{s, a}\right)=\frac{P_{s, a}}{\Delta x} \hat{\mathbf{n}}\left(\mathbf{y}_{s, a}\right)
$$

where $\mathbf{b}_{f \rightarrow s}$ is the body force representing the effect of fluid on structural deformation, $\hat{\mathbf{n}}\left(\mathbf{y}_{s, a}\right)$ is the unit normal direction vector, $\Delta x$ is the uniform initial node spacing (which is assumed same both for fluid and solid). In Eq.(51), the external volumetric pressure force is defined in the interface normal direction. The flowchart for the implementation of the fluid flow effect on the structure is provided in Fig. 7. 


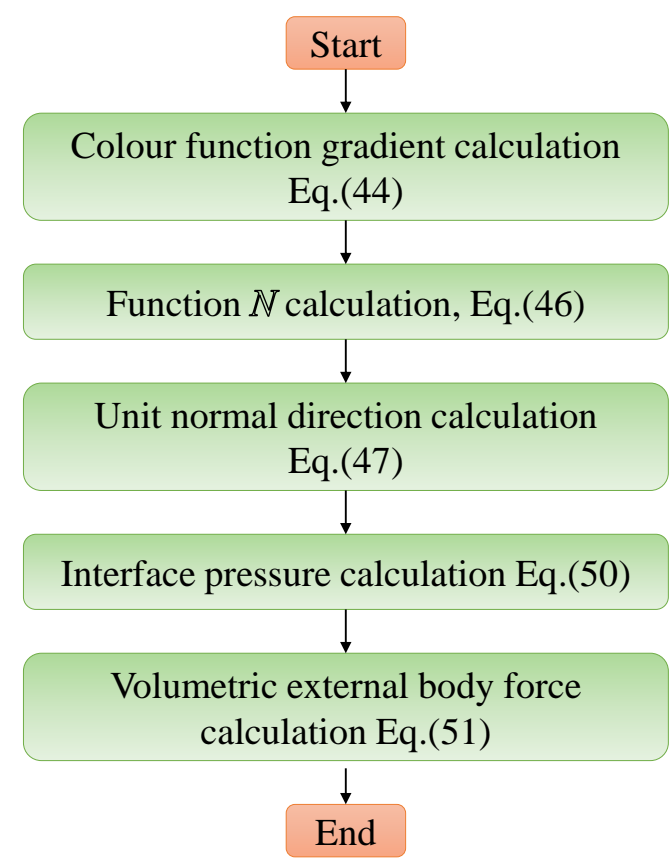

Fig. 7 Flowchart for calculating the effect of fluid force on the solid particle

\subsubsection{Effect of structural deformation on fluid}

The solid in the FSI problems can be categorised as a deformable solid or a rigid solid. In the current FSI coupling model, both solid particles, i.e. either deformable solid particles or rigid solid particles, serve as a boundary condition for fluid flow. Therefore, after finding the structural deformations, the solid particles are treated as the fictitious flow boundary particles for flow simulation. In the present study, the boundary conditions are implemented as proposed in [61]. The viscous force is not included in the pressure calculation for boundary particles, both for non-slip and slip boundary conditions. Only the velocities of the rigid wall particles are calculated differently for non-slip and slip boundary conditions.

Non-slip velocity boundary condition for fluid: the effect of rigid wall

For the non-slip solid boundary condition, the velocity of the rigid wall particle for flow boundary is calculated as [61]

$$
\mathbf{v}_{s, c}=2 \mathbf{v}_{\Gamma}-\left(\sum_{b \in f l u i d} w_{0}\left(\left|\mathbf{y}_{s, c}-\mathbf{y}_{f, b}\right|\right) \mathbf{v}_{f, b}\right)
$$

where $\mathbf{v}_{s, c}$ denotes the velocity of the rigid particle $\mathbf{y}_{s, c}, \mathbf{v}_{\Gamma}$ denotes the prescribed solid wall velocity. $\mathbf{v}_{f, b}$ is the fluid velocity at $\mathbf{y}_{f, b}$. The second term on the right-hand side of Eq.(52) 
is the weighted fluid particle velocity summation. The weighted function is constructed based on the current configuration.

slip velocity boundary condition for fluid: the effect of rigid wall

For the slip boundary condition, the velocity of the rigid particle for flow boundary can be set as

$$
\mathbf{v}_{s, c}=\mathbf{v}_{\Gamma}
$$

Slip and non-slip velocity boundary condition for fluid: the effect of the deformable structure

The velocity of the elastic structural particle $\mathbf{y}_{s, a}$ for flow boundary is set as

$$
\mathbf{v}_{s, a}=\mathbf{v}_{s, a}^{n}
$$

where $\mathbf{v}_{s, a}^{n}$ is obtained from the structural model.

pressure boundary condition for fluid

The effect of fluid pressure acting on flexible solid is calculated by using Eq.(50). The pressure of the flexible solid particles acting on the fluid is also calculated by using Eq.(50) for the conservation of momentum.

The pressure of the rigid solid particles acting on the fluid can be calculated as [61]

$$
P_{s, c}=\frac{\sum_{b \in f \text { fluid }}\left(P_{f, b}+\left(\mathbf{b}_{f, b}-\rho_{f, b} \ddot{\mathbf{u}}_{s, c}\right) \cdot\left(\mathbf{y}_{f, b}-\mathbf{y}_{s, c}\right)\right) w_{0}\left(\left|\mathbf{y}_{f, b}-\mathbf{y}_{s, c}\right|\right) \frac{m_{f, b}}{\rho_{f, b}}}{\sum_{b \in f \text { fluid }} w_{0}\left(\left|\mathbf{y}_{f, b}-\mathbf{y}_{s, c}\right|\right) \frac{m_{f, b}}{\rho_{f, b}}}
$$

where $\mathbf{y}_{s, c}$ is the current position of rigid particle at $\mathbf{x}_{s, c}$.

For the slip boundary condition, the viscous force between the fluid-solid particle interactions is neglected.

\subsection{Numerical procedure}

The numerical procedure is provided in Fig. 8. In the most general fluid-structure interaction problems, there is usually a large difference between the initial configuration and 
the final configuration. Since the OSB-PD theory in the total Lagrangian description is used for the solid model, the solid family members of a solid particle remain the same during the simulations. On the other hand, the PDDO fluid model is defined in the updated Lagrangian description. Thus, the family members of a fluid particle may change in the time integration, including the family members belonging to both solid and fluid regions. Correspondingly, the fluid family members of a solid particle may also change. Consequently, both for a structural particle or for a fluid particle, the family members of it may change with time variation. Therefore, for FSI problems, the configuration updating according to Eq.(22) is performed before the family member array re-construction within each time step.

From Fig. 8, it can also be observed that the density of the fluid particle changes, leading to weakly compressible material property. On the contrary, the density of the structure particle keeps constant during the time integration. Furthermore, the densities of the fluid and the structure may differ from each other. As a result, in the MLS density initialization procedure within the fluid part, when a structure particle is involved in the procedure as a family member of a fluid particle, initial fluid density is used instead of using the solid density. It also should be pointed out that the pressures of the deformable particles are fictitious, they are calculated according to Eq.(50) just for the fluid boundary condition.

Within the time integration, the fluid-structure coupling is achieved by transferring the pressure information from the fluid to the structure as an external body force and transferring the position of the deformed structure particle as a moving boundary condition (shown in dashed arrow). 


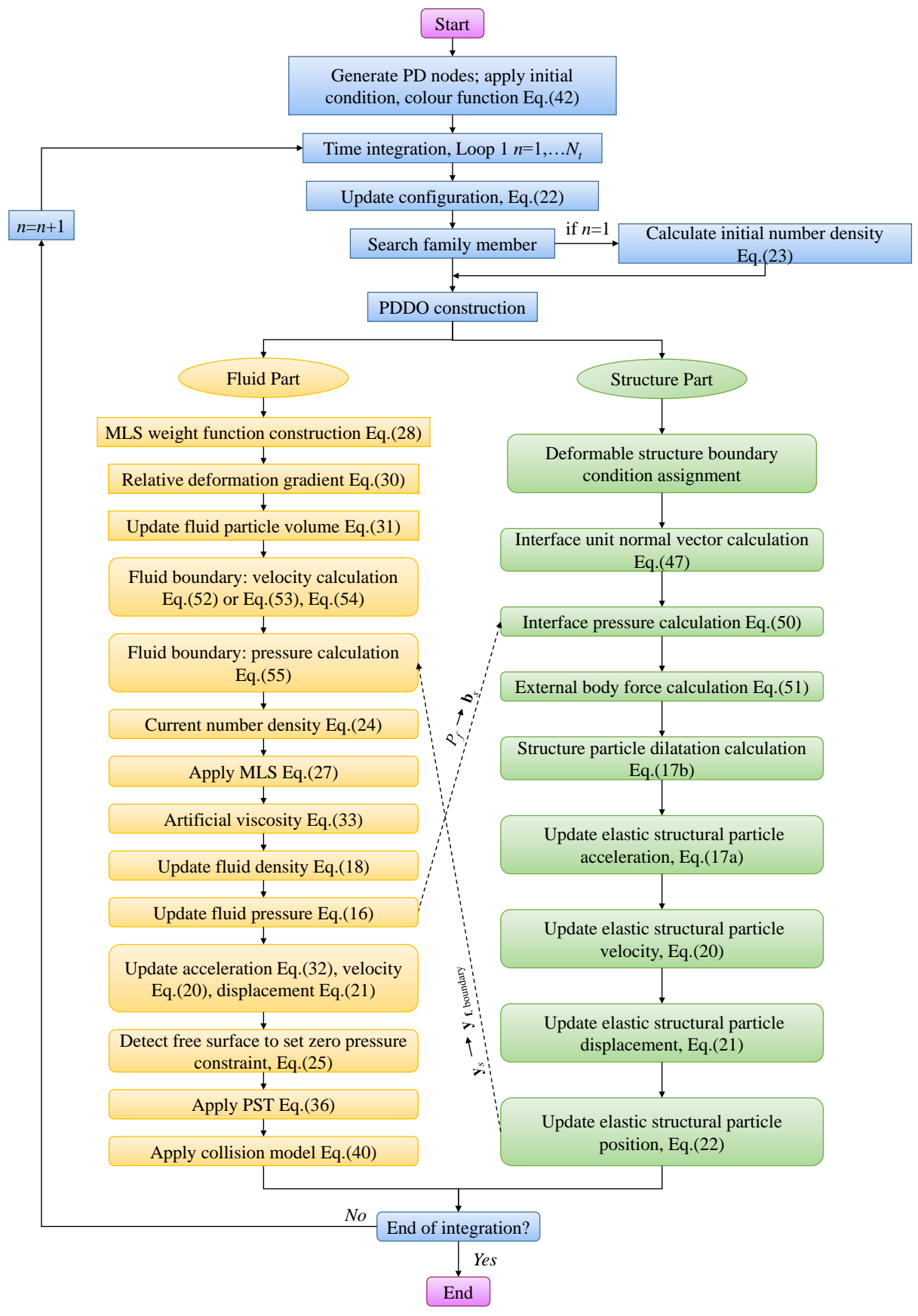

Fig. 8 Numerical procedure

\section{Numerical simulations}

\subsection{Problem description}

In this section, a numerical simulation of a dam collapse under an elastic rubber gate [4, $67,70]$ is conducted to validate the proposed model. The material properties provided in Table 2 both for the fluid and the rubber are set the same as the ones in [4]. The geometry illustration of the problem is provided in Fig. 9 (a). A fluid with dimensions being $L \times H=0.1 \mathrm{~m} \times 0.14 \mathrm{~m}$ 
is within an upper open tank. The bottom, left and upper-right sides of the tank are assumed to be rigid. A rubber gate is located vertically adjacent to the right bottom corner of the fluid column with its dimensions being $0.005 \mathrm{~m} \times 0.079 \mathrm{~m}$. The upper end of the rubber is clamped by the right rigid wall and the bottom end is free to move. The interface between the rubber and fluid is simulated as a non-slip boundary condition, and the boundaries between the fluid and rigid wall are simulated as slip ones. Then under the gravity effect, the fluid will move, and the rubber gate will open and deform under the fluid pressure effect. As to the PD discretization illustrated in Fig. 9 (b), fictitious layers with the thickness being horizon size are added to represent the free surface [34]. These fictitious particles are treated as real fluid particles governed by N-S equation except that their pressures are directly set to be zero.

The boundary conditions are implemented as below:

The interface conditions between the rubber and fluid (non-slip boundary condition):

Effect of fluid flow on the structure:

The effect of fluid pressure acting on the rubber due to fluid flow is calculated by using Eq.(50). Therefore, the volumetric force exerted from the fluid particles on the rubber particle is calculated by using Eq. (51) and it is implemented on the solid particle of the rubber plate.

Effect of structural deformation on the fluid:

The effect of pressure of the rubber particles acting on the fluid flow is calculated by using Eq.(50) and it is implemented on the fluid particles near the rubber plate.

The velocity of the rubber plate boundary is implemented as:

$$
\mathbf{v}_{f, \text { boundary }}=\mathbf{v}_{\text {rubber }}
$$

which is implemented on the fluid particles near the rubber plate.

\section{The boundary conditions on the rigid walls (slip boundary):}

The fluid pressure on the fictitious rigid particles are calculated by using Eq. (55).

The velocities of the fictitious rigid solid particles are implemented as:

$$
\mathbf{v}_{\text {rigid }}=0
$$




\section{The boundary conditions on the free surface:}

The fluid pressure in the fictitious free surface particles are implemented as:

$$
P=0
$$

Table 2 Fluid and rubber material properties

\begin{tabular}{lr}
\hline Parameters & Value \\
\hline Fluid density $\rho_{f}\left(\mathrm{~kg} / \mathrm{m}^{3}\right)$ & 1000 \\
Fluid viscosity $\mu_{f}(\mathrm{~Pa} \cdot \mathrm{s})$ & $1 \times 10^{-3}$ \\
Fluid material constant $\gamma_{f}$ & 7 \\
Rubber density $\rho_{s}\left(\mathrm{~kg} / \mathrm{m}^{3}\right)$ & 1100 \\
Rubber Poisson's ratio $v_{s}$ & 0.4 \\
Rubber elastic modulus $E(\mathrm{MPa})$ & 12.0
\end{tabular}

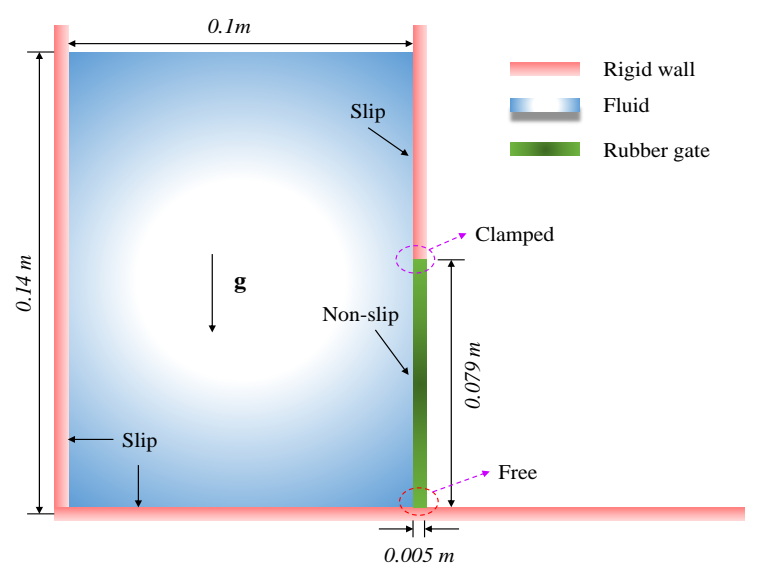

(a)

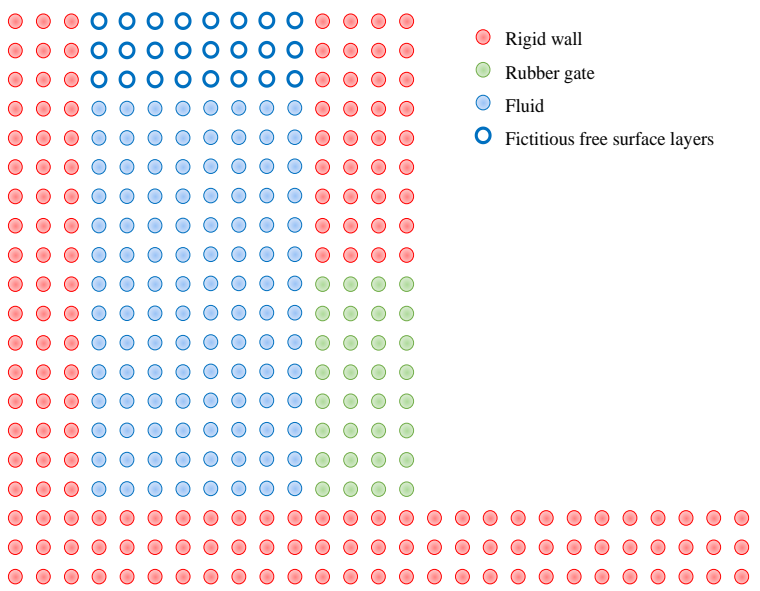

(b)

Fig. 9 (a) Geometry illustration of dam collapse under a rubber gate and (b) PD implementation

The initial particle spacing is set as $\Delta x=0.00125 \mathrm{~m}(80 \times 112$ nodes for fluid). The horizon size is chosen as $\delta=3.6 \Delta x$ to be consistent with one in the MLS scheme. The gravity 
acceleration is set as $|\mathbf{g}|=9.8 \mathrm{~m} / \mathrm{s}^{2}$. The maximum velocity of the fluid is estimated as $\left|\mathbf{v}_{f, \max }\right|=\sqrt{|\mathbf{g}| H}=1.17 \mathrm{~m} / \mathrm{s}$, leading to the artificial speed of sound being $c_{f}=10\left|\mathbf{v}_{f, \max }\right|=11.7 \mathrm{~m} / \mathrm{s}$. The constant time step size is chosen as $\Delta t=1 \times 10^{-5} \mathrm{~s}$ with the total simulation time being $t=0.3 \mathrm{~s}$. The initial damping time is chosen as $t_{\text {damp }}=100 \Delta t$. The MLS scheme is performed every 30 time steps [62]. All the numerical treatments explained in section 4.2 are adopted.

\subsection{Numerical results}

The comparison between the experimental results [4], the numerical results obtained by SPH method [4], and the PD predicted results with corresponding pressure distributions of real fluid particles are provided from Fig. 10 to Fig. 17 for each 0.04s. For the PD simulation results, the particles in blue represent the fluid, the pink particles represent the rigid wall, and the green particles represent the rubber gate. It can be inferred from this qualitative comparison that both the fluid motion and the rubber deformation are consistent from these three sources.

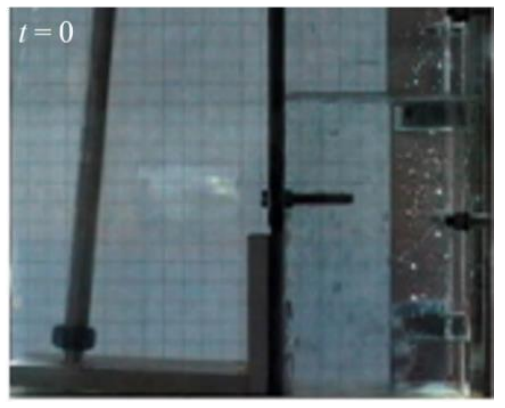

(a)

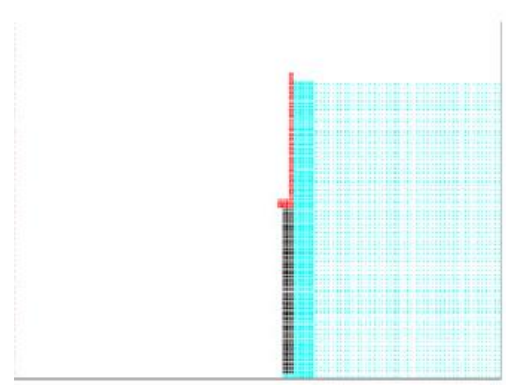

(b)

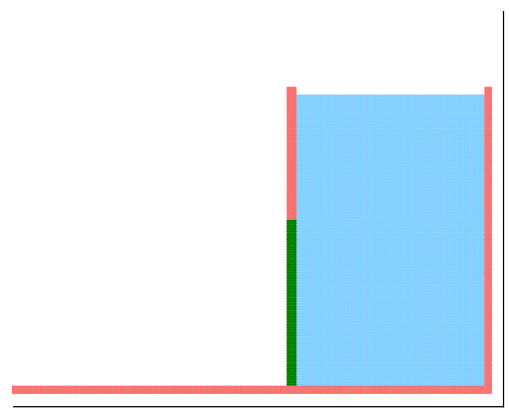

(c)

Fig. 10 Comparison between (a) the experiment image [4], (b) SPH results [4], and (c) PD at $t=0$

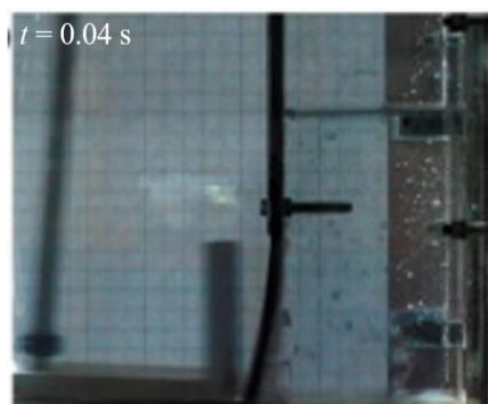

(a)

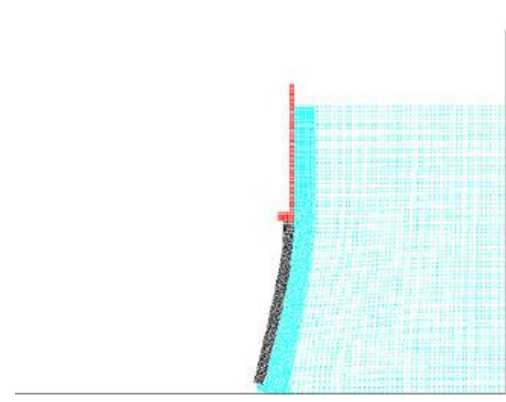

(b)

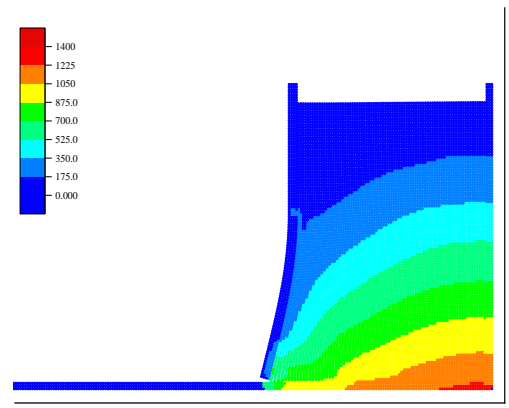

(c)

Fig. 11 Comparison between (a) the experiment image [4], (b) SPH results [4], and (c) PD pressure distribution $(\mathrm{Pa})$ at $t=0.04 \mathrm{~s}$ 


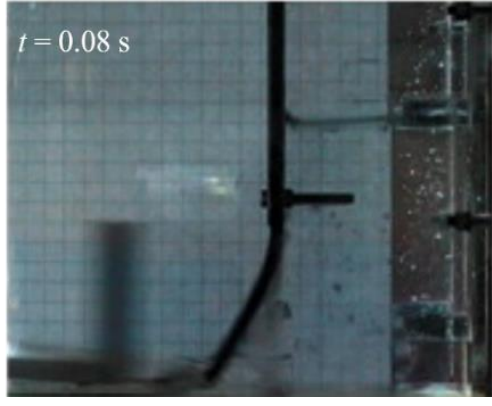

(a)

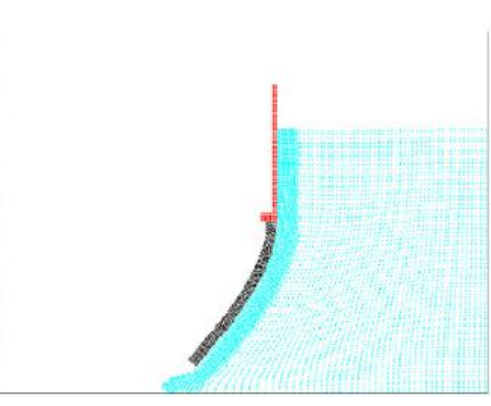

(b)

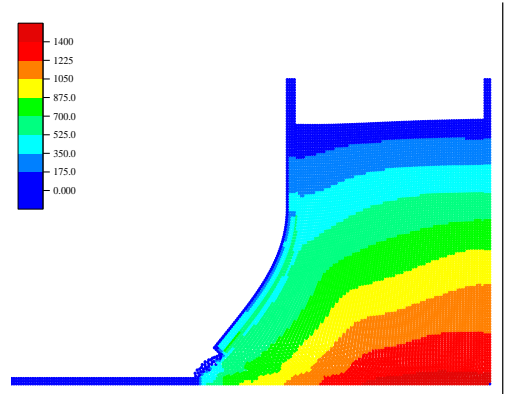

(c)

Fig. 12 Comparison between (a) the experiment image [4], (b) SPH results [4], and (c) PD pressure distribution $(\mathrm{Pa})$ at $t=0.08 \mathrm{~s}$

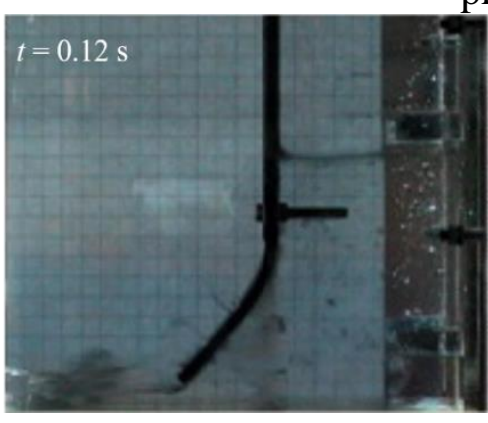

(a)

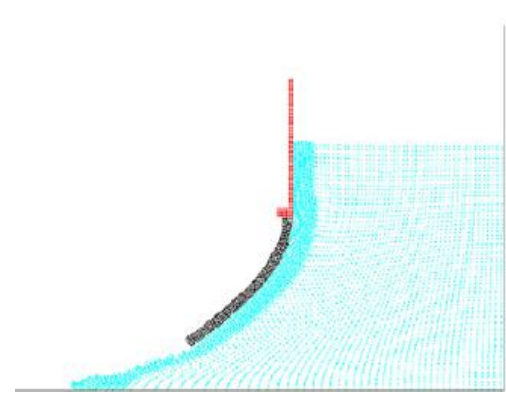

(b)

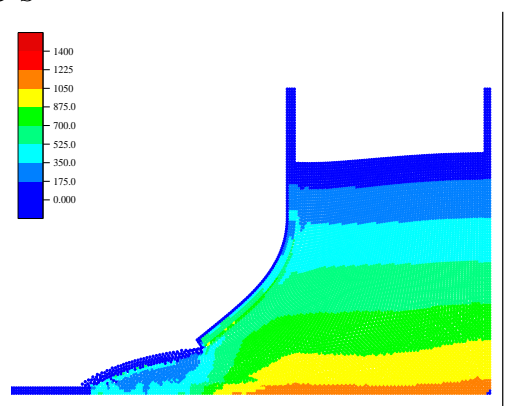

(c)

Fig. 13 Comparison between (a) the experiment image [4], (b) SPH results [4], and (c) PD pressure distribution $(\mathrm{Pa})$ at $t=0.12 \mathrm{~s}$

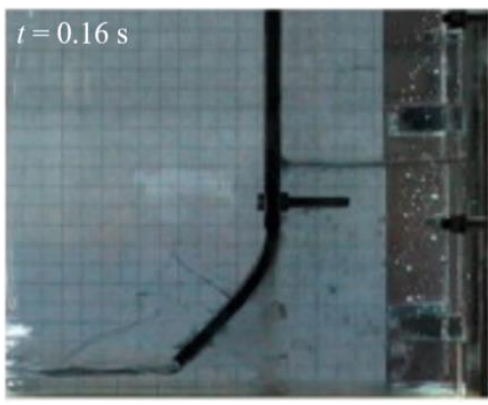

(a)

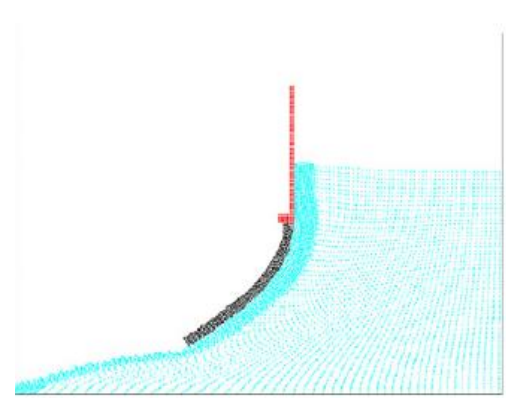

(b)

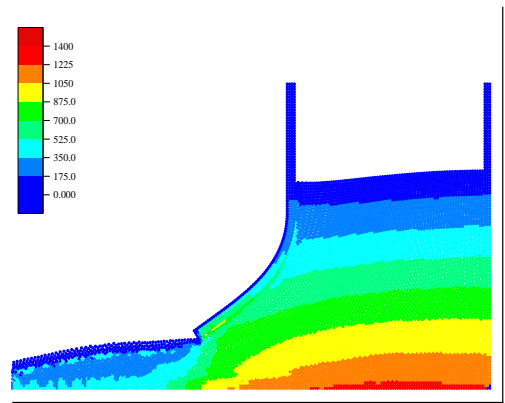

(c)

Fig. 14 Comparison between (a) the experiment image [4], (b) SPH results [4], and (c) PD pressure distribution $(\mathrm{Pa})$ at $t=0.16 \mathrm{~s}$ 


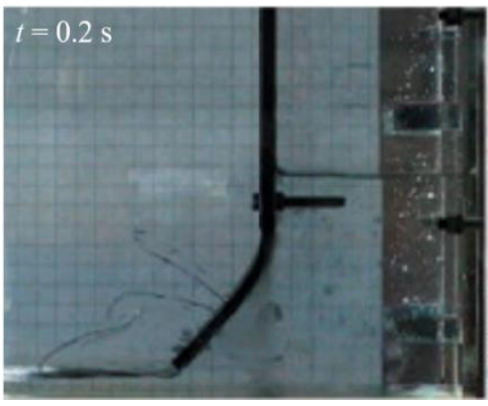

(a)

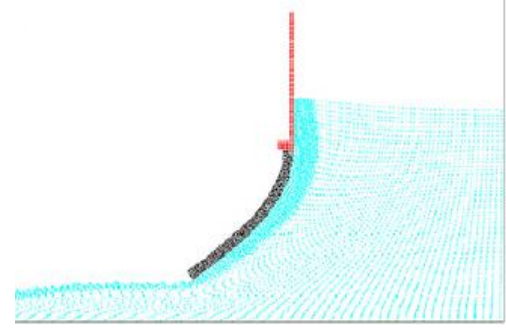

(b)

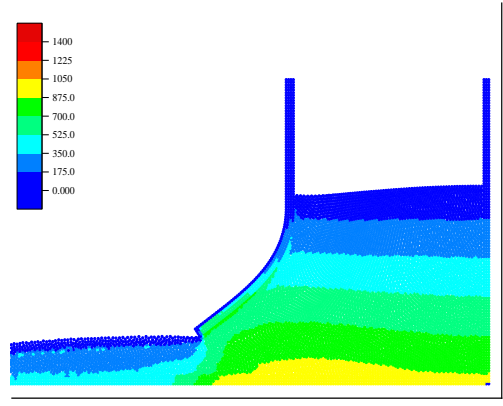

(c)

Fig. 15 Comparison between (a) the experiment image [4], (b) SPH results [4], and (c) PD pressure distribution $(\mathrm{Pa})$ at $t=0.2 \mathrm{~s}$

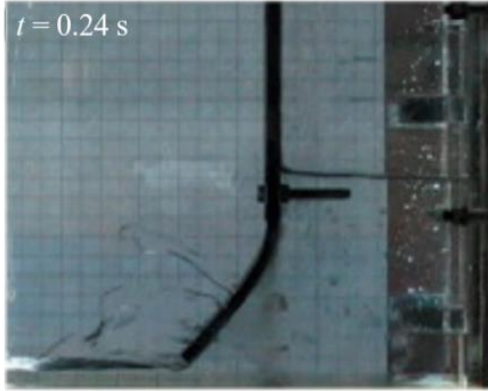

(a)

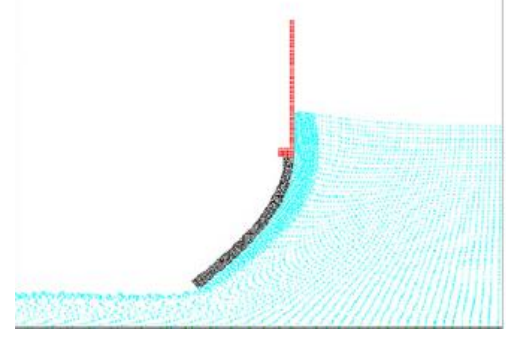

(b)

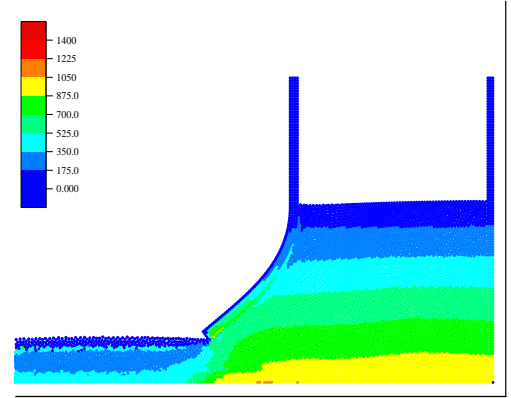

(c)

Fig. 16 Comparison between (a) the experiment image [4], (b) SPH results [4], and (c) PD pressure distribution $(\mathrm{Pa})$ at $t=0.24 \mathrm{~s}$

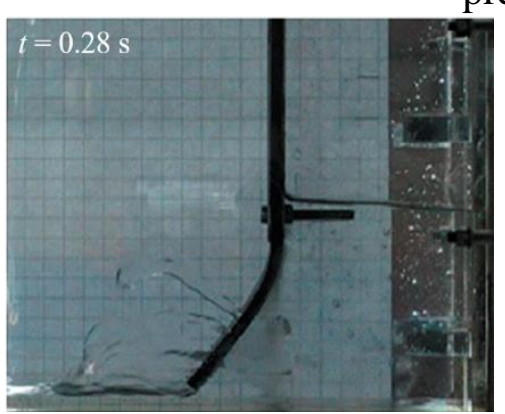

(a)

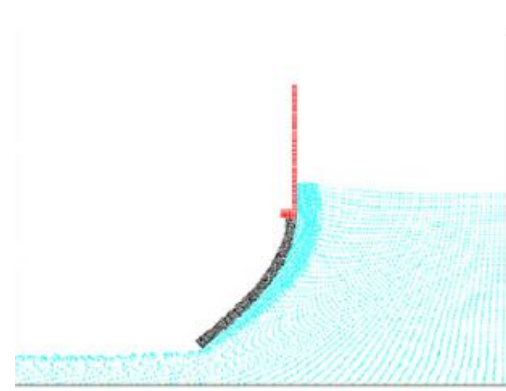

(b)

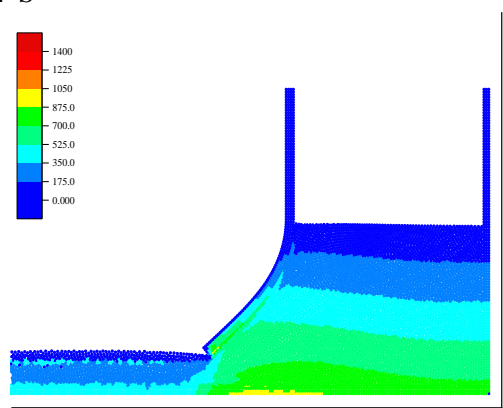

(c)

Fig. 17 Comparison between (a) the experiment image [4], (b) SPH results [4], and (c) PD pressure distribution $(\mathrm{Pa})$ at $t=0.28 \mathrm{~s}$

The rubber gate is initiated to open due to the pressure of the stored water, leading to the water flowing out of the gate. Then with the decreasing of the water level behind the rubber gate, the water pressure acting on the rubber becomes smaller and smaller. Hence, the rubber gate gradually moves back under the resultant force of the gravity force and the water pressure.

A quantitative comparison is also performed to validate the proposed PD model. The simulation results from the resolutions $40 \times 56,60 \times 84$, and $80 \times 112$ are compared. The time history of the displacement of the free end of the plate, the water level near the rigid wall, and the water level with its distance from the rubber gate being $d=0.05 \mathrm{~m}$ are provided in Fig. 18 
and Fig. 19. Besides, the data obtained from the experiment [4] and other numerical methods $[4,14,67,71]$ are presented for comparison.

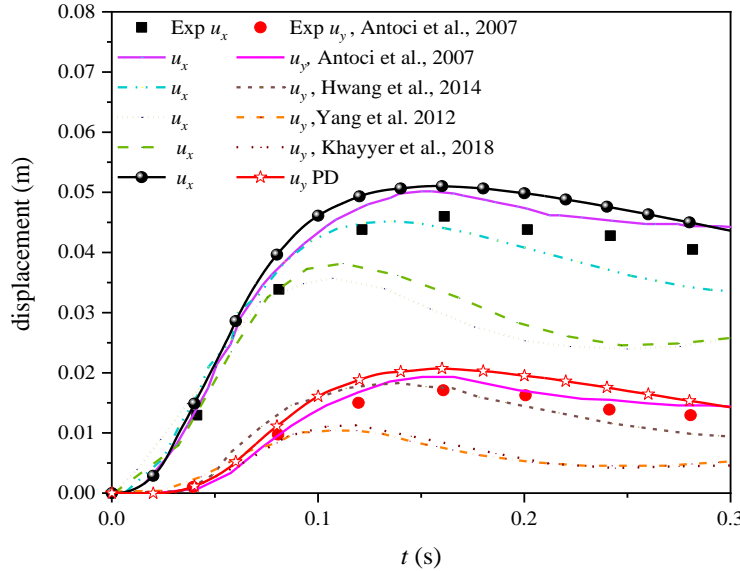

(a)

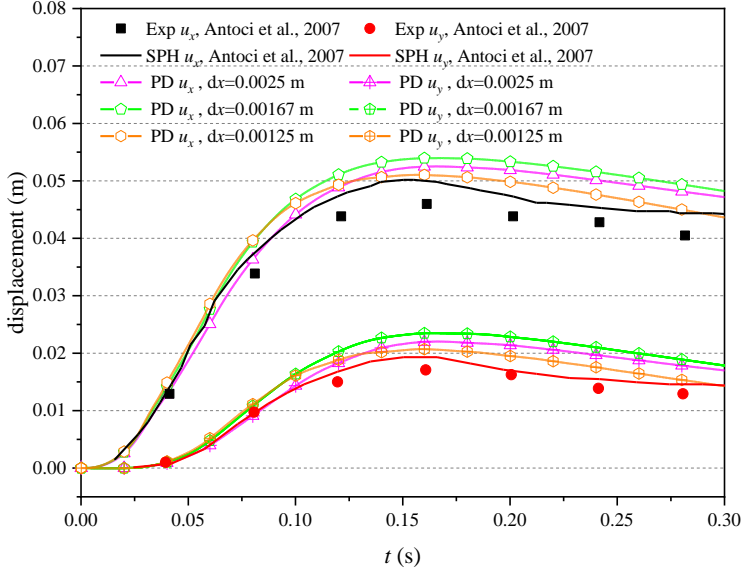

(b)

Fig. 18 (a) Comparison for horizontal and vertical displacements (m) of the free end of the plate between the experiment results [4], SPH simulation result by Antoci et al. [4], MPS simulation result by Hwang et al. [14], Khayyer et al.[71], SPH-FEM simulation results by Yang et al. [67], and PD simulation results, (b) convergence study

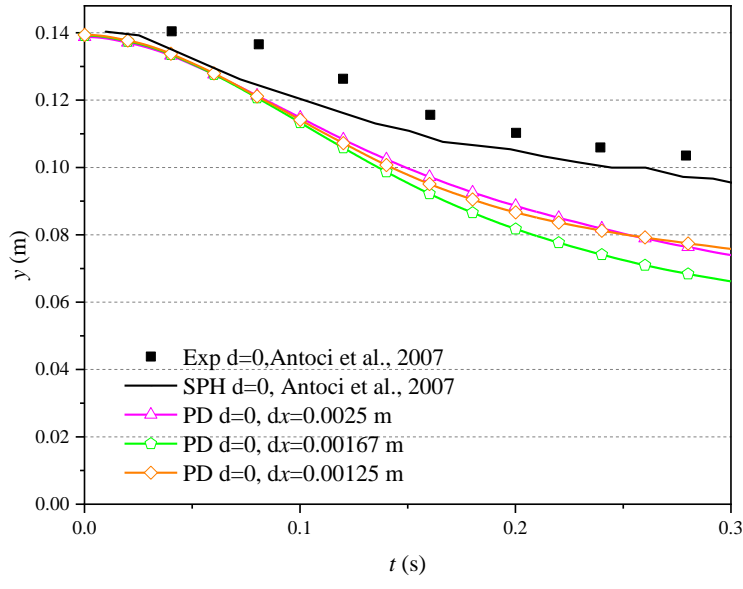

(a)

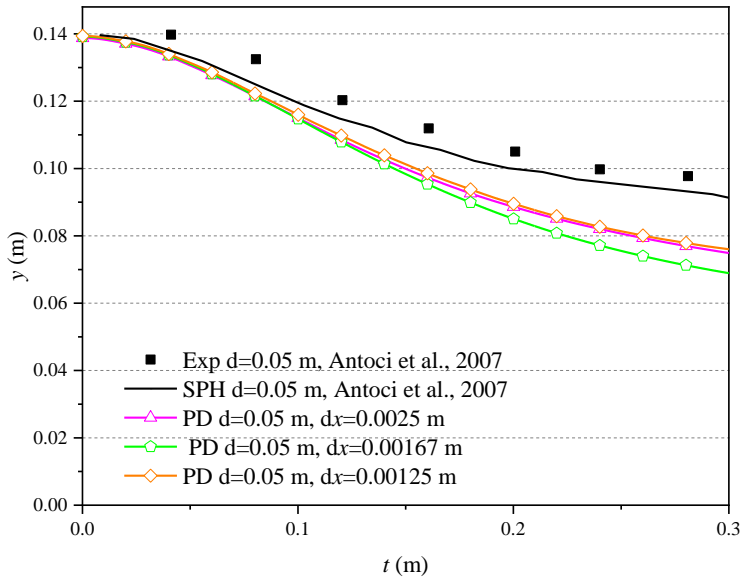

(b)

Fig. 19 Water level (m) comparison just behind the gate (a) and $5 \mathrm{~cm}$ far from it (b) between the experiment results [4], SPH simulation result [4], and PD simulation results

It can be observed from Fig. 18, the displacements of the free end of the rubber gate increase until $t=0.16 \mathrm{~s}$, which consistent with the observation from the qualitative comparison. Then after this peak point, the displacements both in the horizontal and vertical directions decrease until the end of the simulation, indicating the moving back of the rubber gate to its initial shape. By analysing the time history of the water levels from the two locations 
( $d=0$ and $d=5 \mathrm{~cm}$ ), the conclusion can be drawn that the velocity of the fluid volume increasing outside the rubber gate at the end of the simulation becomes much smaller than the one in the early stage. This is due to the smaller opening distance between the rubber gate and the bottom rigid plate compared with the one in the early stage. The quantitative study here agrees well with the previous qualitative study. In conclusion, from the quantitative comparison presented in Fig. 18 and Fig. 19, the current PD model is validated through the good agreement between the PD simulation results and the results from the experiment and SPH method.

However, the discrepancies can also be observed both from PD and other numerical techniques. By investigating the sensitivity of the numerical parameters and techniques, the discrepancies may come from the following aspects. First, the PD node density may affect the accuracy of the final simulation results. In this case, the bending moment of the rubber gate acts an important role in the deformation process. For the rubber PD model, only 4 nodes exist in the thickness direction, indicating that all the 4 nodes experience truncated integration domains. Therefore, the surface correction approach [16] which can improve the accuracy of PD simulations, especially for the particles near the surface, can be utilized. In this study, a simple surface correction is adopted in Eq.(1) as $s_{c}\left(\mathbf{x}_{s, i}\right)=\left(\pi h_{t h i c k} \delta_{s}^{2}\right) / \sum_{j=1, j \in \Omega_{s}}^{N_{i}} V_{s, j}$. By comparing the PD predicted results from the aforementioned different cases in Fig. 18-Fig. 19, refining the PD nodes can increase the accuracy. Second, the application of the MLS and PST provided in Section 4.2, can make the current approach more stable but they may decrease the accuracy. For example, by comparing the time history of the displacement of the free rubber gate end, it can be observed that the application of the PST enlarges the discrepancy between the PD and experiment results. The PST applied in the current study does not have any modification regarding the free surface, which may decrease the accuracy of the numerical simulation [72]. The modifications of PST on free surfaces [73, 74] can be further incorporated into the present model to improve the accuracy. Furthermore, the discrepancy of the water height between the PD predicted results and experimental results may due to the application of fictitious free surface layers. The figure is plotted based on the real fluid particles, not the exactly free surface particles, thus the predicted water height is lower than the real one since fictitious free surface layers are added in the simulation.

The pressure and the velocity snapshots at the final state of $t=0.3 \mathrm{~s}$ are provided in Fig. 20. The pressure and velocity predicted by the PD approach present a similar distribution 
compared by the results provided in $[14,67]$. It should be mentioned that in Fig. 20 (a), the pressure of the rubber represents the fictitious solid pressure calculated according to Eq.(50) for boundary implementation, they are not from the constitutive equations of the solid rubber.

Regarding the fluid pressure, the pressure inside the dam is similar to hydrostatic pressure distribution. When only the free surface detecting approach provided in Section 4.2.1 is adopted, the fluid pressure outside the rubber gate may all become zero because of the over predicted number of free surface particles. The utilization of the PST in the current study can overcome this problem, which largely improves the accuracy of the pressure distribution of the fluid which is outside the rubber gate.

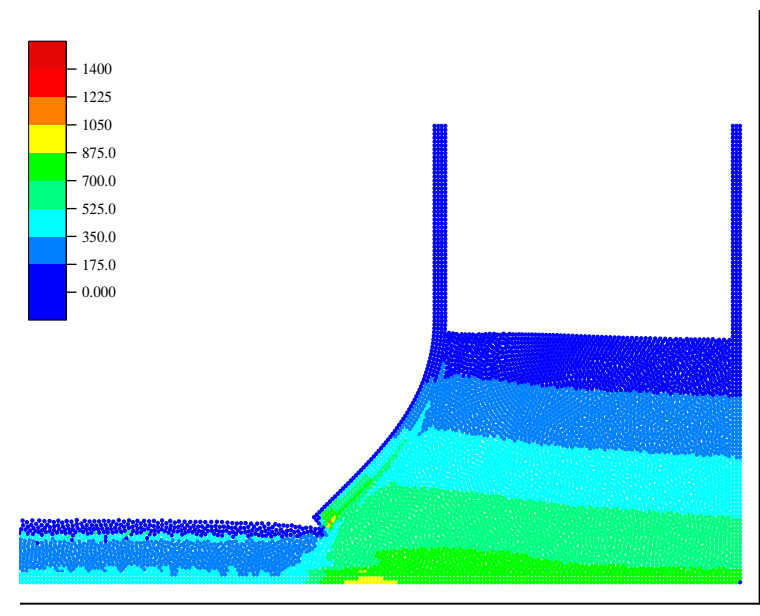

(a)

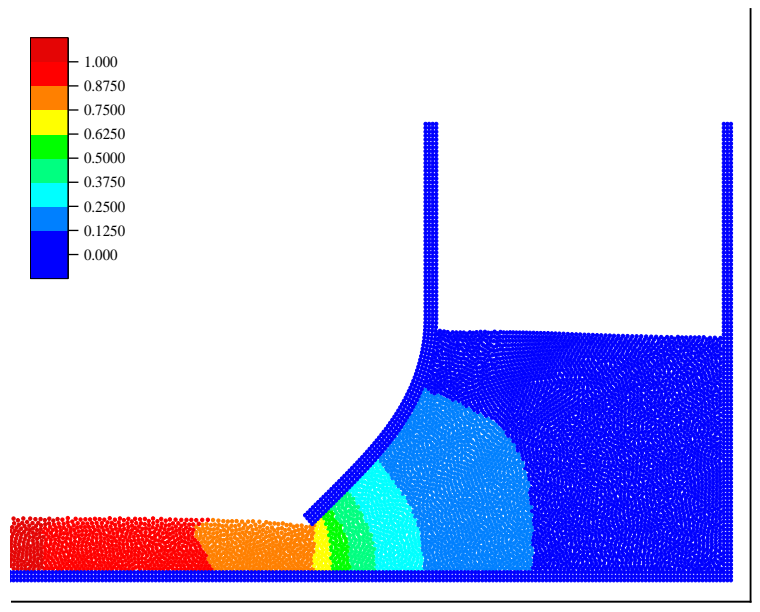

(b)

Fig. 20 PD simulation results (a) Pressure and (b) velocity magnitude at the final state of $t=0.3 \mathrm{~s}$

The configuration at $t=0.3 \mathrm{~s}$ and its close view of the fluid-structure interface are provided in Fig. 21. The unit normal vector of the solid particles for the FSI interface is presented in Fig. 21 (b). The good agreement between the unit normal vector direction and the geometry indicates the capability of the proposed PD approach for accurately predicting the FSI interface. The interface normal direction approach provided in [4] has the limitation that it cannot be applied to the corner particles. However, the unit normal vectors of boundary rubber particles in the present study are also well represented because of the non-local property of the PD theory and the adoption of the colour function. Thus the aforementioned limitation in [4] is removed. In addition, it can also be observed in Fig. 21 (b) that no particle penetration occurs in the FSI interface region. Hence, the boundary collision model proposed in Section 4.2.7 is validated which has positive effects on preventing the particle penetration. 


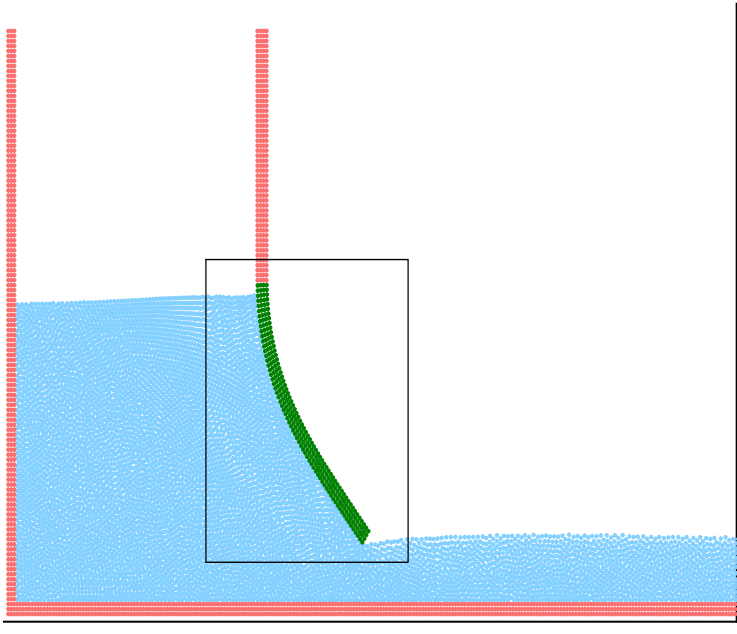

(a)

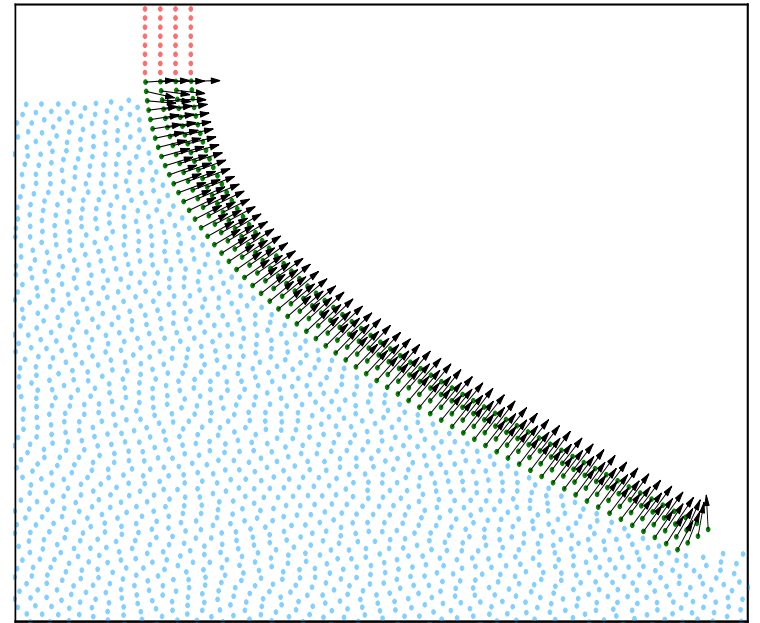

(b)

Fig. 21 PD simulation results (a) configuration and (b) zoom view of the FSI interface state of $t=0.3 \mathrm{~s}$

\section{Conclusion}

In this study, a peridynamic based methodology for fluid-structure interaction problems is proposed. The structural model is developed by using the ordinary state-based peridynamic theory and the fluid model is developed by the peridynamic differential operator. The bidirectional coupling scheme is adopted.

As a demonstration, dam collapse under an elastic rubber gate is simulated by using the newly developed model. The PD predicted results are compared with the experimental and other numerical simulation methods. The good agreement demonstrates the capability of the current model for fluid-elastic structure interaction problems.

As the first PD model using the OSB-PD model for solid and PDDO model for fluid, the current study provides an alternative way to solve fluid-structure interaction problems. Besides, because of the non-local property of PD theory, the numerical implementation of FSI interface is easier.

\section{Acknowledgement}

The authors gratefully acknowledge financial support from the China Scholarship Council (CSC No. 201506230126) and University of Strathclyde.

\section{References}

[1] Bazilevs Y, Takizawa K, Tezduyar TE. Computational fluid-structure interaction: methods and applications: John Wiley \& Sons; 2013. 
[2] Cao XY, Ming FR, Zhang AM. Sloshing in a rectangular tank based on SPH simulation. Appl Ocean Res 2014;47: 241-54.

[3] Faltinsen OM. Hydroelastic slamming. Journal of Marine Science and Technology 2001;5(2): 49-65.

[4] Antoci C, Gallati M, Sibilla S. Numerical simulation of fluid-structure interaction by SPH. Computers \& Structures 2007;85(11-14): 879-90.

[5] Souli M, Ouahsine A, Lewin L. ALE formulation for fluid-structure interaction problems. Computer Methods in Applied Mechanics and Engineering 2000;190(5-7): 659-75.

[6] Zhu L, Peskin CS. Simulation of a Flapping Flexible Filament in a Flowing Soap Film by the Immersed Boundary Method. Journal of Computational Physics 2002;179(2): 452-68.

[7] Paredes RJ, Imas L. Application of multiphase SPH to fluid structure interaction problems. 9th international SPHERIC workshop CNAM Paris, France2014.

[8] Sun PN, Le Touzé D, Zhang AM. Study of a complex fluid-structure dam-breaking benchmark problem using a multi-phase SPH method with APR. Engineering Analysis with Boundary Elements 2019;104: 240-58.

[9] Khayyer A, Gotoh H, Falahaty H, Shimizu Y. An enhanced ISPH-SPH coupled method for simulation of incompressible fluid-elastic structure interactions. Computer Physics Communications 2018;232: 139-64.

[10] Khayyer A, Gotoh H, Shimizu Y. Comparative study on accuracy and conservation properties of two particle regularization schemes and proposal of an optimized particle shifting scheme in ISPH context. Journal of Computational Physics 2017;332: 236-56.

[11] Liu M, Zhang Z. Smoothed particle hydrodynamics (SPH) for modeling fluid-structure interactions. Science China Physics, Mechanics \& Astronomy 2019;62(8): 984701.

[12] Wu K, Yang D, Wright N. A coupled SPH-DEM model for fluid-structure interaction problems with free-surface flow and structural failure. Computers \& Structures 2016;177: 14161.

[13] Khayyer A, Tsuruta N, Shimizu Y, Gotoh H. Multi-resolution MPS for incompressible fluid-elastic structure interactions in ocean engineering. Appl Ocean Res 2019;82: 397-414.

[14] Hwang S-C, Khayyer A, Gotoh H, Park J-C. Development of a fully Lagrangian MPSbased coupled method for simulation of fluid-structure interaction problems. Journal of Fluids and Structures 2014;50: 497-511.

[15] Silling SA. Reformulation of elasticity theory for discontinuities and long-range forces. Journal of the Mechanics and Physics of Solids 2000;48(1): 175-209.

[16] Madenci E, Oterkus E. Peridynamic theory and its applications: Springer; 2014.

[17] Silling SA, Epton M, Weckner O, Xu J, Askari E. Peridynamic States and Constitutive Modeling. Journal of Elasticity 2007;88(2): 151-84.

[18] Oterkus E. Peridynamic theory for modeling three-dimensional damage growth in metallic and composite structures: University of Arizona; 2010.

[19] Oterkus E, Madenci E. Peridynamic Theory for Damage Initiation and Growth in Composite Laminate. Key Engineering Materials 2011;488-489: 355-58.

[20] Oterkus E, Madenci E. Peridynamics for Failure Prediction in Composites. 53rd AIAA/ASME/ASCE/AHS/ASC Structures, Structural Dynamics and Materials Conference: American Institute of Aeronautics and Astronautics; 2012.

[21] Bobaru F, Wang Y, Zhang G. Dynamic effects in unidirectional fiber-reinforced composites: a peridynamic analysis. Computational Modeling of Fracture and Failure 2015: 133.

[22] Diyaroglu C, Oterkus E, Madenci E, Rabczuk T, Siddiq A. Peridynamic modeling of composite laminates under explosive loading. Composite Structures 2016;144: 14-23. 
[23] Madenci E, Oterkus S. Peridynamic modeling of thermo-oxidative damage evolution in a composite lamina. 58th AIAA/ASCE/AHS/ASC Structures, Structural Dynamics, and Materials Conference2017. 0197.

[24] Gao Y, Oterkus S. Thermomechanical Analysis of Composites Under Shock Load Using Peridynamics. Proc. The 28th International Ocean and Polar Engineering Conference International Society of Offshore and Polar Engineers 2018;8.

[25] Gao Y, Oterkus S. Fully coupled thermomechanical analysis of laminated composites by using ordinary state based peridynamic theory. Composite Structures 2019;207: 397-424.

[26] Oterkus E, Madenci E. Peridynamic analysis of fiber-reinforced composite materials. Journal of Mechanics of Materials and Structures 2012;7(1): 45-84.

[27] Oterkus S, Madenci E. Peridynamic modeling of fuel pellet cracking. Engineering Fracture Mechanics 2017;176: 23-37.

[28] Oterkus S, Madenci E, Agwai A. Fully coupled peridynamic thermomechanics. Journal of the Mechanics and Physics of Solids 2014;64: 1-23.

[29] Gao Y, Oterkus S. Ordinary state-based peridynamic modelling for fully coupled thermoelastic problems. Continuum Mechanics and Thermodynamics 2018;31(4): 907-37.

[30] Oterkus S, Madenci E. Peridynamics for fully coupled thermomechanical analysis of fiber reinforced laminates. 55th AIAA/ASMe/ASCE/AHS/sc structures, structural dynamics, and materials conference2014. 0694.

[31] Alpay S, Madenci E. Crack Growth Prediction in Fully-Coupled Thermal and Deformation Fields Using Peridynamic Theory. 54th AIAA/ASME/ASCE/AHS/ASC Structures, Structural Dynamics, and Materials Conference2013. 1477.

[32] Nguyen CT, Oterkus S. Peridynamics for the thermomechanical behavior of shell structures. Engineering Fracture Mechanics 2019;219: 106623.

[33] Madenci E, Barut A, Futch M. Peridynamic differential operator and its applications. Computer Methods in Applied Mechanics and Engineering 2016;304: 408-51.

[34] Gao Y, Oterkus S. Nonlocal numerical simulation of low Reynolds number laminar fluid motion by using peridynamic differential operator. Ocean Eng 2019;179: 135-58.

[35] Gao Y, Oterkus S. Non-local modeling for fluid flow coupled with heat transfer by using peridynamic differential operator. Engineering Analysis with Boundary Elements 2019;105: 104-21.

[36] Oterkus S, Madenci E, Oterkus E. Fully coupled poroelastic peridynamic formulation for fluid-filled fractures. Eng Geol 2017;225: 19-28.

[37] Ouchi H, Katiyar A, Foster JT, Sharma MM. A peridynamics model for the propagation of hydraulic fractures in naturally fractured reservoirs. SPE Journal 2017;22(04): 1,082-1,102. [38] Dalla Barba F, Campagnari P, Zaccariotto M, Galvanetto U, Picano F. A Fluid-Structure Interaction model based on Peridynamics and Navier-Stokes equations for hydraulic fracture problems. 6th European Conference on Computational Mechanics (ECCM 6), 7th European Conference on Computational Fluid Dynamics (ECFD 7). Glasgow, UK2018.

[39] Liu R, Yan J, Li S. Modeling and simulation of ice-water interactions by coupling peridynamics with updated Lagrangian particle hydrodynamics. Computational Particle Mechanics 2019;7(2): 241-55.

[40] Vazic B, Oterkus E, Oterkus S. Peridynamic approach for modelling ice-structure interactions. Trends in the Analysis and Design of Marine Structures: Proceedings of the 7th International Conference on Marine Structures (MARSTRUCT 2019, Dubrovnik, Croatia, 6-8 May 2019): CRC Press; 2019. 55.

[41] Lu W, Li M, Vazic B, Oterkus S, Oterkus E, Wang Q. Peridynamic modelling of fracture in polycrystalline ice. Journal of Mechanics 2019.

[42] Vazic B, Oterkus E, Oterkus S. In-plane and out-of plane failure of an ice sheet using peridynamics. Journal of Mechanics 2019: 1-7. 
[43] Tu Q, Li S. An updated Lagrangian particle hydrodynamics (ULPH) for Newtonian fluids. Journal of Computational Physics 2017;348: 493-513.

[44] Kilic B, Madenci E. An adaptive dynamic relaxation method for quasi-static simulations using the peridynamic theory. Theoretical and Applied Fracture Mechanics 2010;53(3): 194204.

[45] Oterkus S. Peridynamics for the solution of multiphysics problems [Doctor of Philosophy]. Arizona: The University of Arizona; 2015.

[46] Monaghan JJ. Smoothed Particle Hydrodynamics. Annu Rev Astron Astr 1992;30(1): 543-74.

[47] Shao S, Lo EYM. Incompressible SPH method for simulating Newtonian and nonNewtonian flows with a free surface. Advances in Water Resources 2003;26(7): 787-800.

[48] Gomez-Gesteira M, Rogers BD, Dalrymple RA, Crespo AJC. State-of-the-art of classical SPH for free-surface flows. Journal of Hydraulic Research 2010;48(sup1): 6-27.

[49] Sitaraman H, Grout R. An adaptive timestepping methodology for particle advance in coupled CFD-DEM simulations. arXiv preprint arXiv:180209579 2018.

[50] Di Renzo A, Di Maio FP. Comparison of contact-force models for the simulation of collisions in DEM-based granular flow codes. Chemical Engineering Science 2004;59(3): 52541.

[51] Bhandari A, Han J. Investigation of geotextile-soil interaction under a cyclic vertical load using the discrete element method. Geotextiles and Geomembranes 2010;28(1): 33-43.

[52] Jajcevic D, Siegmann E, Radeke C, Khinast JG. Large-scale CFD-DEM simulations of fluidized granular systems. Chemical Engineering Science 2013;98: 298-310.

[53] Koshizuka S, Oka Y. Moving-Particle Semi-Implicit Method for Fragmentation of Incompressible Fluid. Nucl Sci Eng 1996;123(3): 421-34.

[54] Li G, Gao J, Wen P, Zhao Q, Wang J, Yan J, et al. A review on MPS method developments and applications in nuclear engineering. Computer Methods in Applied Mechanics and Engineering 2020;367: 113166.

[55] Lee B-H, Park J-C, Kim M-H, Hwang S-C. Step-by-step improvement of MPS method in simulating violent free-surface motions and impact-loads. Computer Methods in Applied Mechanics and Engineering 2011;200(9): 1113-25.

[56] Macdonald JR. Some simple isothermal equations of state. Reviews of Modern Physics 1966;38(4): 669.

[57] Monaghan JJ, Rafiee A. A simple SPH algorithm for multi-fluid flow with high density ratios. Int J Numer Meth Fl 2013;71(5): 537-61.

[58] Sloper KS, Dourmashkin RR, Bird RB, Slavin G, Webster ADB. Chronic Malabsorption Due to Cryptosporidiosis in a Child with Immunoglobulin Deficiency. Gut 1982;23(1): 80-82.

[59] Koshizuka S, Nobe A, Oka Y. Numerical analysis of breaking waves using the moving particle semi-implicit method. Int J Numer Meth Fl 1998;26(7): 751-69.

[60] Liu M-b, Liu G-r. Smoothed Particle Hydrodynamics (SPH): an Overview and Recent Developments. Archives of Computational Methods in Engineering 2010;17(1): 25-76.

[61] Adami S, Hu XY, Adams NA. A generalized wall boundary condition for smoothed particle hydrodynamics. Journal of Computational Physics 2012;231(21): 7057-75.

[62] Colagrossi A, Landrini M. Numerical simulation of interfacial flows by smoothed particle hydrodynamics. Journal of Computational Physics 2003;191(2): 448-75.

[63] Madenci E. Peridynamic integrals for strain invariants of homogeneous deformation. ZAMM - Journal of Applied Mathematics and Mechanics/Zeitschrift für Angewandte Mathematik und Mechanik 2017.

[64] Suresh P, Kumar SSP, Pantaik BSV. A Comparative Study of Two Different Density Estimation Techniques for Multi-Phase Flow Simulations Using SPH. International Journal for Computational Methods in Engineering Science and Mechanics 2019;20(1): 29-47. 
[65] Shibata K, Masaie I, Kondo M, Murotani K, Koshizuka S. Improved pressure calculation for the moving particle semi-implicit method. Computational Particle Mechanics 2015;2(1): 91-108.

[66] Xu R, Stansby P, Laurence D. Accuracy and stability in incompressible SPH (ISPH) based on the projection method and a new approach. Journal of Computational Physics 2009;228(18): 6703-25.

[67] Yang Q, Jones V, McCue L. Free-surface flow interactions with deformable structures using an SPH-FEM model. Ocean Eng 2012;55: 136-47.

[68] Hirt CW, Nichols BD. Volume of fluid (VOF) method for the dynamics of free boundaries. Journal of Computational Physics 1981;39(1): 201-25.

[69] Morris JP. Simulating surface tension with smoothed particle hydrodynamics. Int J Numer Meth Fl 2000;33(3): 333-53.

[70] Liu M-b, Shao J-r, Li H-q. Numerical simulation of hydro-elastic problems with smoothed particle hydrodynamics method. Journal of Hydrodynamics 2013;25(5): 673-82.

[71] Khayyer A, Gotoh H, Falahaty H, Shimizu Y. Towards development of enhanced fullyLagrangian mesh-free computational methods for fluid-structure interaction. Journal of Hydrodynamics 2018;30(1): 49-61.

[72] Akbari H. An improved particle shifting technique for incompressible smoothed particle hydrodynamics methods. Int J Numer Meth Fl 2019;90(12): 603-31.

[73] Zhang ZL, Walayat K, Huang C, Chang JZ, Liu MB. A finite particle method with particle shifting technique for modeling particulate flows with thermal convection. International Journal of Heat and Mass Transfer 2019;128: 1245-62.

[74] Lind SJ, Xu R, Stansby PK, Rogers BD. Incompressible smoothed particle hydrodynamics for free-surface flows: A generalised diffusion-based algorithm for stability and validations for impulsive flows and propagating waves. Journal of Computational Physics 2012;231(4): 1499523.

\section{Appendix A: PD form of relative deformation gradient in updated Lagrangian description}

The PD form of deformation gradient in total Lagrangian description for 2D problems is $[17,63]$

$$
\mathbf{F}=\frac{2}{\pi h_{\text {thick }} \delta_{f}^{4}} \int_{H_{\mathbf{x}}}\left(\frac{\delta}{\left|\mathbf{x}_{f}^{\prime}-\mathbf{x}_{f}\right|}\right)^{2}\left(\mathbf{y}_{f}^{\prime}-\mathbf{y}_{f}\right) \otimes\left(\mathbf{x}_{f}^{\prime}-\mathbf{x}_{f}\right) \mathrm{d} V_{f}^{\prime}
$$

where $\mathbf{x}_{f}$ is the point of interest and $\mathbf{x}_{f}^{\prime}$ is its family member. The positions of point $\mathbf{x}_{f}$ and $\mathbf{x}_{f}^{\prime}$ in the updated configuration are denoted as $\mathbf{y}_{f}$ and $\mathbf{y}_{f}^{\prime}$, respectively. The discretised form of Eq.(A.1) is expressed as

$$
\mathbf{F}=\frac{2}{\pi h_{\text {thick }} \delta_{f}^{4}} \sum_{j=1}^{N_{i}}\left(\frac{\delta_{f}}{\left|\mathbf{x}_{f, j}-\mathbf{x}_{f, i}\right|}\right)^{2}\left(\mathbf{y}_{f, j}-\mathbf{y}_{f, i}\right) \otimes\left(\mathbf{x}_{f, j}-\mathbf{x}_{f, i}\right) V_{f, j}
$$


where $\mathbf{x}_{f, i}$ and $\mathbf{x}_{f, j}$ are the initial positions of the point of interest $i$ and its family member $j$. The updated positions are denoted by $\mathbf{y}_{f, i}$ and $\mathbf{y}_{f, j}$, respectively. Consequently, $\left(\mathbf{x}_{f, j}-\mathbf{x}_{f, i}\right)$ represents the relative position in the initial configuration which is also the reference configuration in the total Lagrangian description. $\left(\mathbf{y}_{f, j}-\mathbf{y}_{f, i}\right)$ represents the updated relative position.

For the updated Lagrangian description, the current configuration is set as the reference configuration. Therefore, instead of using the initial relative position as Eq. (A.2), the relative position in the current configuration $\left(\mathbf{y}_{f, j}^{n}-\mathbf{y}_{f, i}^{n}\right)$ is used as the reference. Therefore, the deformation gradient Eq. (A.2) for current configuration at time $t_{n}$ in updated Lagrangian description becomes

$$
\mathbf{F}_{n}=\frac{2}{\pi h_{\text {thick }} \delta_{f}^{4}} \sum_{j=1}^{N_{i}}\left(\frac{\delta_{f}}{\left|\mathbf{y}_{f, j}^{n}-\mathbf{y}_{f, i}^{n}\right|}\right)^{2}\left(\mathbf{y}_{f, j}^{n}-\mathbf{y}_{f, i}^{n}\right) \otimes\left(\mathbf{y}_{f, j}^{n}-\mathbf{y}_{f, i}^{n}\right) V_{f, j}^{n}
$$

where $V_{f, j}^{n}$ represents the volume of the point at $\mathbf{y}_{f, j}^{n}$. Similarly, the deformation gradient for updated configuration at time $t_{n+1}$ becomes

$$
\mathbf{F}_{n+1}=\frac{2}{\pi h_{t h i c k} \delta_{f}^{4}} \sum_{j=1}^{N_{i}}\left(\frac{\delta_{f}}{\left|\mathbf{y}_{f, j}^{n}-\mathbf{y}_{f, i}^{n}\right|}\right)^{2}\left(\mathbf{y}_{f, j}^{n+1}-\mathbf{y}_{f, i}^{n+1}\right) \otimes\left(\mathbf{y}_{f, j}^{n}-\mathbf{y}_{f, i}^{n}\right) V_{f, j}
$$

It should be noted that since the updated Lagrangian description is used, the family members of each fluid point are updated every time step. For example, the point at $\mathbf{y}_{f, j}^{n}$ is the family member of the point at $\mathbf{y}_{f, i}^{n}$ with $\left|\mathbf{y}_{f, j}^{n}-\mathbf{y}_{f, i}^{n}\right| \leq \delta_{f}$ at time $t_{n}$. 